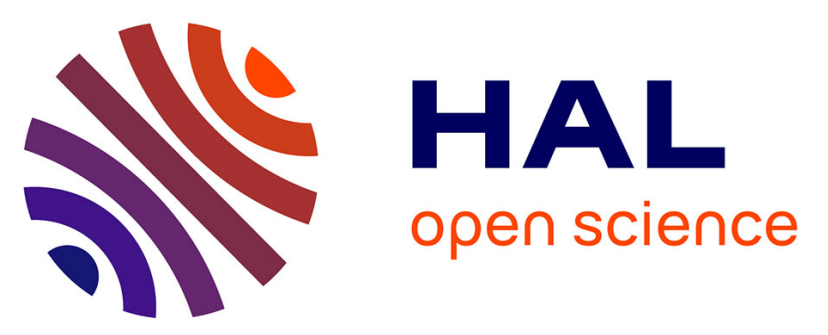

\title{
Modeling of coupled phase transformation and reorientation in shape memory alloys under non-proportional thermomechanical loading
}

Dimitris Chatziathanasiou, Yves Chemisky, Georges Chatzigeorgiou, Fodil

Meraghni

\section{To cite this version:}

Dimitris Chatziathanasiou, Yves Chemisky, Georges Chatzigeorgiou, Fodil Meraghni. Modeling of coupled phase transformation and reorientation in shape memory alloys under nonproportional thermomechanical loading. International Journal of Plasticity, 2016, 82, pp.192-224. 10.1016/j.ijplas.2016.03.005 . hal-01360902

\section{HAL Id: hal-01360902 \\ https://hal.science/hal-01360902}

Submitted on 6 Sep 2016

HAL is a multi-disciplinary open access archive for the deposit and dissemination of scientific research documents, whether they are published or not. The documents may come from teaching and research institutions in France or abroad, or from public or private research centers.
L'archive ouverte pluridisciplinaire HAL, est destinée au dépôt et à la diffusion de documents scientifiques de niveau recherche, publiés ou non, émanant des établissements d'enseignement et de recherche français ou étrangers, des laboratoires publics ou privés. 


\title{
Modeling of coupled phase transformation and reorientation in shape memory alloys under non-proportional thermomechanical loading
}

\author{
Dimitris Chatziathanasiou*, Yves Chemisky, George Chatzigeorgiou, Fodil Meraghni \\ Arts et Métiers Paris Tech, LEM3 UMR 7239 CNRS \\ 4 rue Augustin Fresenl, F-57078 Metz - cedex 01, France
}

\begin{abstract}
In the present study, a new 3D thermodynamic coupled model is proposed for SMAs. The behavior of SMA structures is described through several strain mechanisms, each associated with its proper internal variables. This model is built to capture the particular behavior of SMAs when subjected to complex loading, namely non-proportional thermomechanical loading. To achieve this task, a new approach to describe the martensitic reorientation mechanism has been introduced in conjuction with a new method to account for forward and reverse transformation. Thermomechanical coupling, related to dissipation and latent heat is fully implemented. The validity of the model is demonstrated by comparing experimental results of complex thermomechanical loading paths of SMA structures with numerical simulations.
\end{abstract}

Keywords: Shape memory alloys, Superelasticity, Phase transformation, Reorientation, Non-proportional loading, Numerical simulation

\section{Introduction}

Shape memory alloys (SMAs) are metallic materials named after the discovery of their unique capability to retrieve their original shape when their temperature is increased after a mechanical loading. It is the result of the transformation at the crystallic level between 5 the two key solid phases that the material can adopt, austenite and martensite. The difference between these two phases lies on the architecture of the crystalline structure, which varies between a cubic-like configuration in austenite and a less symmetric configuration in martensite (Patoor et al, 2006, Otsuka and Wayman, 1999). Several different effects have been investigated, for instance superelasticity and actuation, depending on the thermomechanical conditions imposed. Such capabilities rank those materials in the wider class of smart materials, according to their multi-physics (mechanical-thermal) coupling.

\footnotetext{
* Corresponding author

Email: dimitrios.chatziathanasiou@ensam.eu

Preprint submitted to "International Journal of Plasticity"
}

February 13, 2016 
All those effects are based on the fact that such martensitic transformation can take place in both ways, and that the martensitic phase can be reoriented under the action of 15 mechanical forces. The direction from austenite to martensite is systematically defined as forward transformation, whereas the inverse procedure is called reverse transformation. This phase transformation can be the result of a change in temperature between critical values, and/or a change in the mechanical state. In the absence of applied stress, forward transformation occurs between martensite start $\left(M_{s}\right)$ and finish $\left(M_{f}\right)$ temperatures and

20 reverse transformation between austenite start $\left(A_{s}\right)$ and finish $\left(A_{f}\right)$ temperatures. The development of appropriate stress levels can also lead to phase transformation. In particular, applying a mechanical loading/unloading cycle above $A_{f}$ demonstrates the effect of superelasticity in SMAs. During forward transformation, the transformation starts at a critical, temperature-dependent stress. A stress plateau is observed in the uniaxial

25 stress-strain diagram, before the start of the elastic section of martensite. In the case of mechanical loading at temperatures above $A_{f}$, the strain that appears between the two elastic sections on the stress-strain diagram corresponds to a transformation strain. This strain is fully recovered after reverse transformation has finished during unloading.

Martensite is the phase that appears at low temperatures/high stress state and conso sists of zones with different orientation directions found in a single crystal, called variants. Two main forms, distinguished on the basis of the configuration of variants, are observed: twinned martensite, for which the variants appear in multiple directions and form a selfaccommodated assembly ; and detwinned or oriented martensite, for which a principal direction of variants dominates the martensitic composition (Merzouki et al, 2010). Con-

35 trary to the two-way direction of phase transformation, the transition between these two crystallic configurations occurs in one direction, resulting to detwinned martensite only and is called orientation or detwinning. This occurs with the help of mechanical working, when stress is increased between critical levels. Such oriented martensite can still be reoriented if the direction of mechanical forces change. Appropriate combination of

40 orientation and phase transformation processes result in the characteristic shape memory effect (Lagoudas, 2008).

The properties of shape memory and superelasticity render SMAs an interesting material sought to be utilized in practical applications in the last twenty years (Lecce and Concilio, 2014, Barbarino et al, 2014). A significant increase in the interest given to

45 SMAs in publications and patents has recently been observed (Mohd Jani et al, 2014). Specifically, innovative systems were introduced in automotive and aerospace industries (Hartl and Lagoudas, 2007, Van Humbeeck, 1999). SMAs have also found particularly extended use in biomedical applications (Auricchio et al, 2015, Morgan, 2004). This wide array of applications motivates research to develop mathematical models able to

50 capture their particular thermomechanical behavior (Khandelwal and Buravalla, 2009). These models aim at being utilized in robust computational tools, mostly Finite Element Analysis (FEA) methods. Their contribution is associated with the assistance provided to engineers to design SMA actuators and conceive innovative products.

In recent years, various phenomenological models have been proposed to explain 55 the physics behind SMA behavior (Cisse et al, 2015). They focus on the macroscopic variables, allowing for relatively simple numerical implementation with respect to micromechanical approaches based on the physics of the crystalline structure (Patoor and Berveiller, 1997; Lagoudas et al, 2006). The primary macroscopic variable taken in mind in such models is the martensitic volume fraction (MVF). The actual representation 
60 of phase transformation in the macroscopic level is the change of the concentration of martensite in the material, thus justifying this consideration(Hartl et al, 2010, Chemisky et al, 2011, Lexcellent et al, 2006). The direction of strain appearing during transformation is taken in mind using necessarily a tensorial variable that depends on the loading conditions(Luig and Bruhns, 2008). Generally, these models have proven sufficiently ac-

65 curate in capturing the material behavior under unidirectional loading (Peultier et al 2008).

The effect of orientation has also been investigated in recent works (Ameduri et al, 2015, Sedlák et al, 2012, Saleeb et al, 2011; Saint-Sulpice et al, 2009). These models add the feature of simulating three-dimensional loading paths to previous simpler models

70 (Boyd and Lagoudas, 1994; Brinson, 1993, Saleeb et al, 2001). Interesting experimental work has been carried with respect to such loading (Bouvet et al, 2004 Grabe and Bruhns, 2009, Sittner et al, 1995). Reorientation consists in the change of the orientation of the martensite variants in existing martensite volume, without inducing further transformation. The procedure of reorientation has a visible effect on the preferred direction

75 of inelastic strains, whereas detwinning mostly concerns their magnitude (Liu and Favier, 2000, Popov and Lagoudas, 2007). In certain models (Panico and Brinson, 2007, Helm and Haupt, 2003; Arghavani et al, 2010) and subsequent works, two different volume fractions for martensite are considered as driving material properties, one for twinned and one for the detwinned part. This proves a useful consideration, since the evolution

so of the martensitic strain can be associated to thermally induced and stress induced forward transformation. However, most of these models operate under the assumption that there is a direct relation between the stress induced martensitic fraction and an equivalent transformation strain magnitude, as investigated in Souza et al (1998); Juhász et al (2001); Taillard et al (2008).

85 In this paper, a phenomenological model, based on the physical interpretation of the processes that occur inside a SMA polycrystal is developed. The notion of mean transformation strain inside the martensitic volume discussed in the articles of Peultier et al (2008); Chemisky et al (2011) is examined from a macroscopic point of view to redefine the principles of reorientation, forward and reverse transformation. This leads to

9o the introduction of independent scalar rate variables which drive each of the three strain mechanisms. Accordingly, a robust formalism is presented in terms of thermodynamics which is based on a Gibbs free energy potential.

Moreover, the scope of this work extends to providing a general framework for addressing the numerical resolution of multiple strain mechanisms simultaneously activated,

95 allowing for adding even more inelastic strain mechanisms. Motivated by the work of Auricchio et al (2014), each mechanism is thought to have its proper activation criterion. These criteria take the form of yield functions, depending on internal variables. Based on simple observations, the methodology for carrying out the numerical algorithm is presented and the mechanical and thermal tangent moduli are calculated. Furthermore, recognizing the strong coupling of thermomechanical effects on SMA behavior (Peyroux et al, 1998; Morin et al, 2011), the heat caused by mechanical working is calculated under the scope of multiple mechanisms in play. This investigation is able to cover the issue of latent heat which affects mechanical tests in superelasticity (Brinson et al, 2004; Hartl and Lagoudas, 2008).

105 In the first part of this work, a physical description of the three non-linear mechanisms considered is presented. It is followed by the presentation of the thermodynamic 
framework which covers the current model. In the second part, certain important remarks allowing the numerical implementation of the model are given, along with a thermomechanical study. In the third part, results deriving from characteristic numerical implementations are given, demonstrating the validity of the model.

\section{Physical description of deformation mechanisms in Shape Memory Alloys}

Most successful SMA models describe the thermomechanical behavior of such materials with (at least) two important internal variables: The martensitic volume fraction and the transformation stain (Lagoudas, 2008). The derivation of the last quantity as a function of the applied stress has been addressed extensively in the case of transformation only (Qidwai and Lagoudas, 2000). While reorientation of martensitic variants occurs together with a forward or reverse martensitic transformation, the proper derivation of the evolution equation for the transformation strain has to be defined accordingly. Here, a physical representation of the particular behavior of SMAs is described to provide more insight about the derivation of such evolution, depending on the physical deformation mechanisms activated. Its physical and mathematical description constitute the major contribution of the proposed model. However, this description necessitates to entirely review the definition of modeling within a thermodynamical framework, and also imposes the development of a numerical resolution scheme for the strongly coupled resulting set of equations. These two points also constitute major advancements in the simulation of the behavior of SMAs subjected to complex loadings.

\subsection{Deformation mechanisms: Phase transformation and reorientation}

The total continuum of the material is considered divided in a mass of representative volume elements (RVEs). Each of the RVEs has its own continuum for which the proper kinematical relations can be derived. From the work of Chemisky et al. (Chemisky et al 2011), the notion of the mean transformation strain over the martensitic volume fraction of a RVE is already examined. Since it will be an important variable in the formulation of the model, it is chosen here to be the key element in describing the physical representation of the kinematics.

$$
\bar{\varepsilon}^{\boldsymbol{T}}=\frac{1}{V_{M}} \int_{V_{M}} \tilde{\varepsilon}_{r}^{\boldsymbol{T}} d V
$$

In the above expression, $\tilde{\varepsilon}_{r}^{T}$ as the field of transformation strains inside the whole volume $V$ of the RVE for any given point with coordinates $\boldsymbol{r}, V_{M}$ as the martensitic volume in the RVE and $\bar{\varepsilon}^{T}$ is the average value of transformation strain inside $V_{M}$. On the other hand, the total transformation strain of the RVE is considered a variable of the material continuum: it is called here $\varepsilon^{T}$ :

$$
\varepsilon^{T}=\frac{1}{V} \int_{V} \tilde{\varepsilon}_{r}^{T} d V=\frac{1}{V}\left(\int_{V_{A}} \tilde{\varepsilon}_{r}^{T} d V+\int_{V_{M}} \tilde{\varepsilon}_{r}^{T} d V\right)
$$

where $V_{A}$ is the austenitic volume inside the RVE. Knowing that the first integral is zero, and by effect of (1):

$$
\varepsilon^{\boldsymbol{T}}=\frac{V_{M}}{V} \bar{\varepsilon}^{\boldsymbol{T}} \Leftrightarrow \varepsilon^{\boldsymbol{T}}=\xi \bar{\varepsilon}^{\boldsymbol{T}}
$$


$\xi$ being the representation of the martensitic volume fraction (MVF).

The variables used so far correspond to different levels of continua. $\tilde{\varepsilon}_{r}^{T}$ is a local variable. $\xi, \varepsilon^{\boldsymbol{T}}$ and $\bar{\varepsilon}^{\boldsymbol{T}}$ are average values over the of the RVE, corresponding to the material continuum. Each RVE is thus treated as a point of the material continuum, achieving the transition to macroscopical considerations.

Note that the macroscopic effective inelastic strain $\varepsilon^{T}$ is here taken as the volume average of the inelastic strain. This is an assumption that holds only if the average stress in both phases is equal, which is adopted here for such a phenomenological model.

Each particular strain mechanism comes in effect in the evolution of the inelastic strain and the MVF. From (2), the time rate formula will be:

$$
\dot{\varepsilon}^{T}=\dot{\xi} \bar{\varepsilon}^{T}+\xi \dot{\bar{\varepsilon}}^{T}
$$
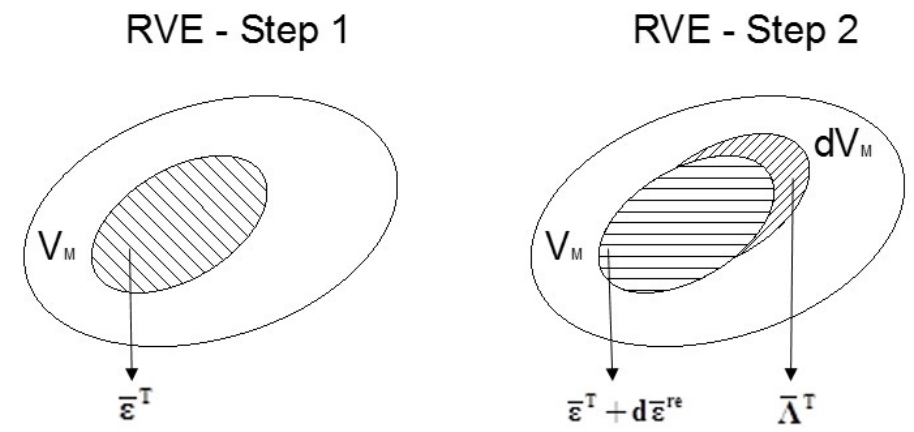

Figure 1: Time step representation of a SMA RVE behavior

An incremental scheme of the RVE of a SMA is studied in Fig. 1. Two consecutive steps in the loading of a RVE are considered, which are observed with a time difference of $d t$. Two mechanisms contribute to the change of $\bar{\varepsilon}^{T}$ : (i) The transformation strain found in the newly added martensitic volume $d V_{M}$, which has a mean value $\overline{\boldsymbol{\Lambda}}^{\boldsymbol{T}}$; (ii) The

140 change of transformation strain in the martensitic volume that already exists, causing a change $\boldsymbol{d} \bar{\varepsilon}^{r e}$;

Here, it is assumed that these two contributions are the effect of two mechanisms physically separated and independent. The first is the direct effect of the martensitic transformation: in the case of forward transformation, it results to the addition of martensitic

145 volume and, if this volume contains detwinned material, to the addition of the corresponding transformation strain. Accordingly, in the case of reverse transformation, it results to the suppression of martensitic volume and the corresponding transformation strain. Forward and reverse transformation do not affect the transformation strains within the preexisting martensitic volume, and therefore are considered independent of reorientation. The second is the direct effect of reorientation and takes place only inside the pre-existing martensitic volume. It can appear without any change of the martensitic volume fraction, and therefore is considered independent of transformation.

The value of the mean transformation strain in the second step will be a weighted average between the contributions of the two mechanisms, based on the volume in which 
they occur. The mean transformation strain in the second step is thus considered equal to:

and the respective differential is:

$$
\frac{\left(\bar{\varepsilon}^{\boldsymbol{T}}+\boldsymbol{d} \bar{\varepsilon}^{r \boldsymbol{e}}\right) V_{M}+\overline{\boldsymbol{\Lambda}}^{\boldsymbol{T}} d V_{M}}{V_{M}+d V_{M}}
$$

$$
\boldsymbol{d} \bar{\varepsilon}^{\boldsymbol{T}}=\frac{\left(\bar{\varepsilon}^{\boldsymbol{T}}+\boldsymbol{d} \bar{\varepsilon}^{r \boldsymbol{e}}\right) V_{M}+\bar{\Lambda}^{\boldsymbol{T}} d V_{M}}{V_{M}+d V_{M}}-\bar{\varepsilon}^{\boldsymbol{T}} \Rightarrow \boldsymbol{d} \bar{\varepsilon}^{\boldsymbol{T}}=\frac{\left(\overline{\boldsymbol{\Lambda}}^{\boldsymbol{T}}-\bar{\varepsilon}^{\boldsymbol{T}}\right) d V_{M}+V_{M} \boldsymbol{d} \bar{\varepsilon}^{r \boldsymbol{e}}}{V_{M}+d V_{M}}
$$

Here, it is assumed that:

$$
d V_{M} \ll V_{M}
$$

which is acceptable in the scope of differential calculus. Thus:

$$
\boldsymbol{d} \bar{\varepsilon}^{\boldsymbol{T}}=\frac{\left(\overline{\boldsymbol{\Lambda}}^{\boldsymbol{T}}-\bar{\varepsilon}^{\boldsymbol{T}}\right) d V_{M}+V_{M} \boldsymbol{d} \bar{\varepsilon}^{r \boldsymbol{e}}}{V_{M}}=\left(\overline{\boldsymbol{\Lambda}}^{\boldsymbol{T}}-\bar{\varepsilon}^{\boldsymbol{T}}\right) \frac{d V_{M}}{V_{M}}+\boldsymbol{d} \bar{\varepsilon}^{r \boldsymbol{e}}
$$

To simplify the term $\frac{d V_{M}}{V_{M}}$, the differential $d \xi$ is considered as:

$$
d \xi=d\left(\frac{V_{M}}{V}\right)=\frac{d V_{M}}{V}-\frac{V_{M}}{V^{2}} d V
$$

The martensitic transformation is considered to be an isochoric process in the sense that

$$
\operatorname{tr}\left(\boldsymbol{d} \varepsilon^{\boldsymbol{T}}\right)=0
$$

(Patoor et al, 1995) and therefore it is recognized that:

$$
d V=V \operatorname{tr}(\boldsymbol{d} \varepsilon)=V \operatorname{tr}\left(\boldsymbol{d} \varepsilon^{e l}+\boldsymbol{d} \varepsilon^{t h}\right)
$$

The contribution of the elastic and thermal expansion of the volume of the RVE could be taken in mind to the full extent. However, for the sake of simplicity, these contributions are considered negligible. As a consequence:

$$
d \xi=\frac{d V_{M}}{V}
$$

which is used to write:

$$
d \xi=\frac{d V_{M}}{V_{M}} \frac{V_{M}}{V}=\frac{d V_{M}}{V_{M}} \xi \Leftrightarrow \frac{d V_{M}}{V_{M}}=\frac{d \xi}{\xi}
$$

Under the light of $(10)$, the equation $(6)$ is written:

$$
\boldsymbol{d} \bar{\varepsilon}^{\boldsymbol{T}}=\left(\bar{\Lambda}^{\boldsymbol{T}}-\bar{\varepsilon}^{\boldsymbol{T}}\right) \frac{d \xi}{\xi}+\boldsymbol{d} \bar{\varepsilon}^{r e}
$$

The rate $\dot{\bar{\varepsilon}}^{r e}$ is viewed as the foremost effect of reorientation: it is the mean rate of change of transformation strains inside a martensitic volume which is considered constant in time (in two consecutive increments):

$$
\dot{\bar{\varepsilon}}^{r e}=\frac{1}{V_{M}} \int_{V_{M}} \dot{\varepsilon}^{T}(r) d V
$$


After giving the definitions for transformation and reorientation, it is considered important to clarify the term "transformation strain". In the scope of this article, it is used to describe the sum of all inelastic strains in the continuum. All non-thermoelastic strain is included into the term transformation strain. It is stressed that this inelastic strain is not the direct product of only the phase transformation itself; it is also affected by reorientation. In this sense, both transformation (either forward or reverse) and reorientation contribute in the evolution of transformation strain. Still, this strain is linked to the lattice transformation/orientation in the martensitic, that is "transformed", volume.

The above description does not in fact constitute a mathematical demonstration of the form of the transformation strain, involving average quantities over subdomains with moving boundaries. Such description is given to present a physical insight in the selection of the averaging quantities and the pragmatic significance of the final form 12 A mathematical formulation of the evolution equation for transformation strain is given in Annex A.
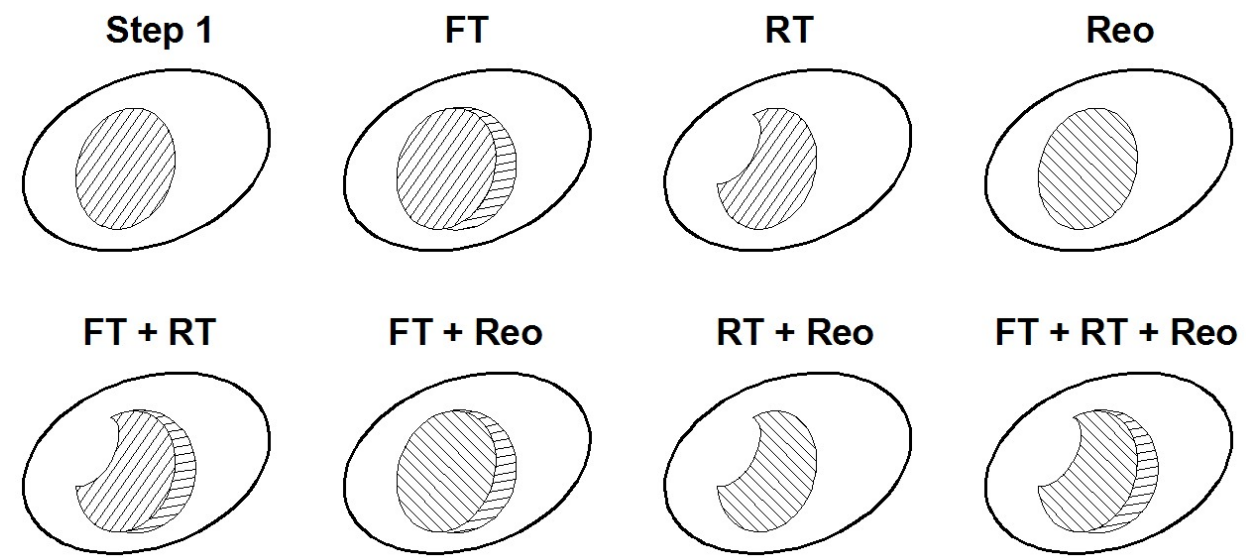

Figure 2: Effect of inelastic mechanisms on a SMA RVE

\subsection{Macroscopic coupling of phase transformation and reorientation strains}

The macroscopic effects of the activation of different strain mechanisms on a RVE are visualized in Fig. 2. The areas with the filled pattern correspond to martensitic volume, whereas the void areas represent the austenitic volume. All the possible combinations are visualized and compared with the starting configuration of the initial step. The orientation of the filled pattern represents the direction of transformation strains inside the martensitic fraction. When reorientation is activated, this orientation changes. During forward transformation it is visible that the added martensitic volume does not necessarily follow the existing strain orientation. Reverse transformation induces the removal of a piece of martensitic volume and along with it the associated transformation strain.

In the macroscopical level, three rate variables are introduced, each of those representing the activation and the magnitude of the effect of their respective mechanisms. $\dot{\xi}^{F}$ represents the rate of change of the MVF induced by forward transformation is acti- 
vated. It is defined by:

$$
\dot{\xi}^{F}=\frac{\dot{V}_{M}^{F}}{V}
$$

$\dot{\xi}^{R}$ represents the rate of change of the MVF induced by reverse transformation is activated:

$$
\dot{\xi}^{R}=\frac{\dot{V}_{M}^{R}}{V}
$$

$\dot{V}_{M}^{F}$ is the increment rate produced by the addition of martensitic volume when forward transformation occurs. Respectively, $\dot{V}_{M}^{R}$ is the increment rate produced by the suppression of martensitic volume during reverse transformation.

The third rate variable $\dot{\bar{p}}^{r e}$ represents the rate of change of the magnitude of the mean transformation strain inside a martensitic part of constant volume. It is defined as:

$$
\dot{\bar{p}}^{r e}=\sqrt{\frac{2}{3} \dot{\bar{\varepsilon}}^{r e}: \dot{\bar{\varepsilon}}^{r e}}
$$

Accordingly,

$$
\dot{\bar{\varepsilon}}^{r e}=\dot{\bar{p}}^{r e} \boldsymbol{\Lambda}_{\varepsilon}^{r e}
$$

where $\Lambda_{\varepsilon}^{r e}$ is a tensor named reorientation tensor representing the direction for the increment of the mean transformation strain induced by reorientation.

Following these definitions, it is necessary that each of these three variables are zero when the respective mechanism is not activated and positive when activated. As for the rate of martensitic volume fraction, it is deduced to take the form of:

$$
\dot{\xi}=\dot{\xi}^{F}-\dot{\xi}^{R}
$$

Indeed, the expression

$$
\dot{\xi}=\frac{\dot{V}_{M}^{F}}{V}-\frac{\dot{V}_{M}^{R}}{V}
$$

complies with the suggestion made in equation (9), where $d V_{M}$ can take positive values for forward and negative values for reverse transformation. It is directly implied that at any given moment,

$$
\xi=\int_{0}^{t} \dot{\xi}^{F} d \tau-\int_{0}^{t} \dot{\xi}^{R} d \tau
$$

These time integrals are denoted as:

$$
\xi^{F}=\int_{0}^{t} \dot{\xi}^{F} d \tau
$$

and

$$
\xi^{R}=\int_{0}^{t} \dot{\xi}^{R} d \tau
$$


In order to comply with the physical limitation of the notion of a volume fraction, it is necessary that:

$$
0 \leq \xi \leq 1 \Leftrightarrow \xi^{R} \leq \xi^{F} \leq 1+\xi^{R}
$$

Furthermore, the effect of each mechanism in the rate of transformation strain is assumed to follow a linear relation with its respective rate variable. The rate of the total transformation strain is partitioned into three contributors, each driven by the different mechanisms:

$$
\dot{\varepsilon}^{T}=\dot{\varepsilon}^{F}+\dot{\varepsilon}^{R}+\dot{\varepsilon}^{r e}
$$

For forward transformation:

$$
\dot{\varepsilon}^{F}=\dot{\xi}^{F} \Lambda_{\varepsilon}^{\boldsymbol{F}}
$$

where $\Lambda_{\varepsilon}^{F}$ is defined as the forward transformation tensor and gives the relation between $\dot{\xi}^{F}$ and the magnitude and direction of the induced increment of transformation strain. For reverse transformation:

$$
\dot{\varepsilon}^{R}=\dot{\xi}^{R} \Lambda_{\varepsilon}^{R}
$$

Here, the definition reverse transformation tensor is presented, which gives the relation between $\dot{\xi}^{F}$ and the magnitude and direction of the induced increment of transformation strain. As already established at the beginning of the subsection, this should be:

$$
\Lambda_{\varepsilon}^{R}=-\bar{\varepsilon}^{T}
$$

For reorientation, the effect of the rate of increment of mean transformation strain within the martensitic part contributes to the rate of transformation strain in the RVE proportionally to its fraction:

$$
\dot{\varepsilon}^{r e}=\xi \dot{\bar{\varepsilon}}^{r e}
$$

and, following equation 16 :

$$
\dot{\varepsilon}^{r e}=\xi \dot{\bar{p}}^{r e} \boldsymbol{\Lambda}_{\varepsilon}^{r e}
$$

Here, the product $\xi \dot{p}^{r e}$ is replaced by the new rate variable $\dot{p}^{r e}$ to reach:

$$
\dot{\varepsilon}^{r e}=\dot{p}^{r e} \Lambda_{\varepsilon}^{r e}
$$

Thus, equation $(22)$ is rewritten:

$$
\dot{\varepsilon}^{\boldsymbol{T}}=\dot{\xi}^{F} \boldsymbol{\Lambda}_{\varepsilon}^{\boldsymbol{F}}+\dot{\xi}^{R} \boldsymbol{\Lambda}_{\varepsilon}^{\boldsymbol{R}}+\dot{p}^{r e} \boldsymbol{\Lambda}_{\varepsilon}^{r e}
$$

An important assumption is implied here: Since forward and reverse transformation are considered to appear independently, they may occur simultaneously. In this case, recalling the definition of $\bar{\Lambda}^{T}$ at A.7 it is deduced that, at any moment:

$$
\dot{\xi}^{F} \boldsymbol{\Lambda}_{\varepsilon}^{\boldsymbol{F}}+\dot{\xi}^{R} \boldsymbol{\Lambda}_{\varepsilon}^{\boldsymbol{R}}=\dot{\xi} \overline{\boldsymbol{\Lambda}}^{\boldsymbol{T}}
$$

A simultaneous activation of forward and reverse transformation that may take place during loading presents interesting effects. The increment of forward transformation strain is thought to follow the direction of evolving stress (see Fig. 2). The exact relation will be examined later. The evolution of stress is not necessary colinear with previous stress states nor with preexisting strain, thus leading to new average strain direction. 
190 An effect of "apparent reorientation" is therefore observed, even if the mechanism of reorientation is activated. A similar phenomenon is proposed in (Saint-Sulpice et al, 2009). In that work, the term reorientation is used to describe the exact effect of coupled forward and reverse transformation. Careful consideration should be taken when referring to this term in our present article. Still, simulations conducted in our work demonstrate

195 that this effect would not be enough to fully capture the change in the direction of inelastic strains and that a full model should be used for complex loading cases.

By using the time integral of equation (22), a partition of the total transformation strain in the contribution of forward and reverse transformation and reorientation is proven evident:

$$
\varepsilon^{T}=\varepsilon^{F}+\varepsilon^{R}+\varepsilon^{r e}
$$

\section{Thermodynamic formulation of the phenomenological model}

Having defined the main variables that are actually representative of the deformation mechanisms described in the previous sections, it is essential to describe hereafter the general framework of thermodynamics in which this work is developed. Also, the proper selection of internal variables, associated to each phenomenon, and the formulation of a thermodynamic potential will lead to the couplings (i.e. the reciprocal impact of the progression of physical mechanisms on each other) that naturally arise during arbitrary complex loadings. Next, criteria for transformation and reorientation are defined from

205 those thermodynamic considerations. Finally, evolution equations are expressed accordingly, taking into account the important features highlighted with respect to reverse transformation (see also Lagoudas et al (2012)).

\subsection{Free energy potential and general thermodynamic forces}

Generally, the macroscopic behavior of SMAs is approached by means of using suitable constitutive equations which involve state variables of the material (Patoor et al, 2006). Thermodynamic state variables are those that represent all quantities that characterize a material body at a certain state (Coleman and Gurtin, 1967). If they can be observed, they are called external state variables, otherwise internal state variables (Lagoudas 2008). Henceforth, the set of all the internal variables will be denoted as $\boldsymbol{V}$.

215 Those constitutive equations are derived through a prescribed thermodynamic potential. This is a function that characterizes a certain thermodynamic state of the body and depends on the state variables. At every state, the thermodynamic potential represents a quantity of energy within the material system. Therefore, it evokes products of the state variables with their thermodynamically conjugant quantities, called the general

220 thermodynamic forces (GTFs). The set of all GTFs will be henceforth denoted as $\boldsymbol{A}$.

It is commonly shown that the transformation strain is thermodynamically conjugant to stress, usually by implementing the procedure first applied by Coleman and Noll (1963) under the conditions described by Lubliner (1972). All the basic laws of continuum mechanics need to be validated through the implementation of the thermodynamic

225 potential, including the second law of thermodynamics, usually expressed by the local form of the Clausius-Duhem inequality (Lemaitre and Chaboche, 2002). 
In the thermodynamic framework, it is aimed to incorporate the assumptions made in section 2. A Gibbs free energy potential is chosen to be the base of our model:

$$
\begin{aligned}
G= & U_{o}-s_{o} T+C_{v}\left[\Delta T-T \ln \left(\frac{T}{T_{o}}\right)\right]-\frac{1}{2} \boldsymbol{\sigma}: S: \boldsymbol{\sigma}-\boldsymbol{\sigma}: \boldsymbol{\alpha} \Delta T-\boldsymbol{\sigma}: \boldsymbol{\varepsilon}^{\mathbf{T}}+ \\
& \left(1+\lambda^{r e}\right) \boldsymbol{X}: \boldsymbol{v}^{\boldsymbol{r e}}+H(\xi)
\end{aligned}
$$

where:

$\boldsymbol{\sigma}$ is the Cauchy stress.

$S$ is the elastic compliance tensor.

230 $\quad \alpha$ is the thermal expansion coefficient.

$\Delta T=T-T_{o}$ is the difference between the current temperature $T$ and the temperature at the reference state $T_{o}$.

$U_{o}$ is the specific internal energy at the reference state.

$s_{o}$ is the specific entropy at the reference state.

${ }_{235} C_{v}$ is the specific heat capacity.

$H(\xi)$ is a function accounting for the isotropic hardening associated with transformation, linked to the martensitic volume fraction (Hartl et al, 2010).

$\boldsymbol{v}^{\boldsymbol{r} \boldsymbol{e}}$ is defined as the hardening strain for reorientation.

$\boldsymbol{X}$ is defined as backstress.

240 Finally, $\lambda^{r e}$ is a limiting cofactor for reorientation.

It is noted that the variables $S, \boldsymbol{\alpha}, U_{o}, s_{o}$ and $C_{v}$ are assumed to vary linearly according to the martensitic volume fraction. For example:

$S=S^{A}+\xi\left(S^{M}-S^{A}\right)$ for the elastic compliance tensor, where the superscripts $A$ and

$245 \quad M$ stand for the austenitic and martensitic phases respectively. Similar assumption has been adopted in Lagoudas et al (2012), supported by the analysis of Boyd and Lagoudas. In the case of mechanical elastic properties, it was shown that using either the rule of mixtures on the compliance tensors (which is assumed here) or a micromechanical scheme (Mori-Tanaka) has little impact on the effective response (Boyd and Lagoudas, 1994).

The internal variables are chosen to be: $\boldsymbol{\sigma}, T, \boldsymbol{\varepsilon}^{\boldsymbol{F}}, \boldsymbol{\varepsilon}^{\boldsymbol{R}}, \boldsymbol{\varepsilon}^{\boldsymbol{r} \boldsymbol{e}}, \boldsymbol{v}^{\boldsymbol{r e}}, \xi^{F^{\prime}}, \xi^{R}$.

Following the typical Coleman-Noll procedure, the entropy $s$ and the total strain $\varepsilon$ are found as:

$$
s=-\frac{\partial G}{\partial T}=s_{o}+\boldsymbol{\sigma}: \boldsymbol{\alpha}+C_{v} \ln \left(\frac{T}{T_{o}}\right)
$$

and

$$
\varepsilon=-\frac{\partial G}{\partial \boldsymbol{\sigma}}=S: \boldsymbol{\sigma}+\boldsymbol{\alpha} \Delta T+\varepsilon^{\mathbf{T}}
$$

In the latter, a typical decomposition of the total strain is recognized:

$$
\varepsilon=\varepsilon^{\mathrm{el}}+\varepsilon^{\mathrm{th}}+\varepsilon^{\mathrm{T}}
$$

250 where $\varepsilon^{\mathrm{el}}=S: \sigma$ is the elastic and $\varepsilon^{\text {th }}=\boldsymbol{\alpha} \Delta T$ is the thermal strain. 
The rest of the derived GTFs of the internal variables are found as:

$$
\begin{aligned}
\boldsymbol{A}_{\varepsilon^{F}}= & -\frac{\partial G}{\partial \varepsilon^{\boldsymbol{F}}}=-\frac{\partial \varepsilon^{\boldsymbol{T}}}{\partial \varepsilon^{\boldsymbol{F}}}: \frac{\partial G}{\partial \varepsilon^{\boldsymbol{T}}}=\boldsymbol{\sigma} \\
\boldsymbol{A}_{\boldsymbol{\varepsilon}^{R}}= & \boldsymbol{A}_{\boldsymbol{\varepsilon}^{r \boldsymbol{e}}}=\boldsymbol{\sigma} \\
\boldsymbol{A}_{\boldsymbol{v}^{r e}}= & -\frac{\partial G}{\partial \boldsymbol{v}^{\boldsymbol{r e}}}=-\left(1+\lambda^{r e}\right) \boldsymbol{X} \\
A_{\xi^{F}}= & -\frac{\partial G}{\partial \xi^{F}}=-\frac{\partial G}{\partial \xi} \frac{\partial \xi}{\partial \xi^{F}}=-\frac{\partial G}{\partial \xi}=-\tilde{U}_{o}+\tilde{s} T-\tilde{C}_{v}\left[\Delta T-T \ln \left(\frac{T}{T_{o}}\right)\right]+ \\
& \frac{1}{2} \boldsymbol{\sigma}: \tilde{\boldsymbol{S}}: \boldsymbol{\sigma}+\boldsymbol{\sigma}: \tilde{\boldsymbol{\alpha}} \Delta T-\frac{\partial \lambda^{r e}}{\partial \xi} \boldsymbol{X}: \boldsymbol{v}^{r \boldsymbol{e}}-\frac{\partial H}{\partial \xi}
\end{aligned}
$$

Here, the variables appearing with a tilde denote the difference of respective constants between the martensitic and austenitic phase. For example:

$\tilde{S}=S^{M}-S^{A}$

Moreover,

$$
\begin{aligned}
& A_{\xi^{R}}=-\frac{\partial G}{\partial \xi} \frac{\partial \xi}{\partial \xi^{R}}=\frac{\partial G}{\partial \xi}=\tilde{U}_{o}-\tilde{s} T+\tilde{C}_{v}\left[\Delta T-T \ln \left(\frac{T}{T_{o}}\right)\right]-\frac{1}{2} \boldsymbol{\sigma}: \tilde{\boldsymbol{S}}: \boldsymbol{\sigma}- \\
& \boldsymbol{\sigma}: \tilde{\boldsymbol{\alpha}} \Delta T+\frac{\partial \lambda^{r e}}{\partial \xi} \boldsymbol{X}: \boldsymbol{v}^{\boldsymbol{r e}}+\frac{\partial H}{\partial \xi}
\end{aligned}
$$

Following Lemaitre and Chaboche (2002), the second thermodynamic law is reduced to:

$$
\boldsymbol{A}_{\boldsymbol{\varepsilon}^{F}}: \dot{\boldsymbol{\varepsilon}}^{\boldsymbol{F}}+A_{\xi^{F}} \dot{\xi}^{F}+\boldsymbol{A}_{\boldsymbol{\varepsilon}^{R}}: \dot{\boldsymbol{\varepsilon}}^{\boldsymbol{R}}+A_{\xi^{R}} \dot{\xi}^{R}+\boldsymbol{A}_{\boldsymbol{\varepsilon}^{r e}}: \dot{\boldsymbol{\varepsilon}}^{r e}+\boldsymbol{A}_{\boldsymbol{v}^{r e}}: \dot{\boldsymbol{v}}^{r \boldsymbol{e}}-\frac{1}{T} \vec{q} \overrightarrow{g r a d} T \geq 0
$$

where $\vec{q}$ is the heat flux and $\overrightarrow{g r a d T}$ the spatial gradient of temperature.

From one hand, the Clausius-Duhem inequality expresses the positive character of these two parts of disspation:

$$
\boldsymbol{A}_{\boldsymbol{\varepsilon}^{F}}: \dot{\boldsymbol{\varepsilon}}^{\boldsymbol{F}}+A_{\xi^{F}} \dot{\xi}^{F}-\boldsymbol{A}_{\varepsilon^{R}}: \dot{\boldsymbol{\varepsilon}}^{\boldsymbol{R}}+A_{\xi^{R}} \dot{\xi}^{R}+\boldsymbol{A}_{\boldsymbol{\varepsilon}^{r e}}: \dot{\boldsymbol{\varepsilon}}^{r e}+\boldsymbol{A}_{\boldsymbol{v}^{r e}}: \dot{\boldsymbol{v}}^{r e} \geq 0
$$

and

$$
-\frac{1}{T} \vec{q} \overrightarrow{\operatorname{grad}} T \geq 0
$$

On the other hand, it is postulated here that the contribution of the internal variables linked to each mechanism to the total dissipation is also non negative:

$$
\left\{\begin{array}{l}
\gamma^{F}=\boldsymbol{A}_{\boldsymbol{\varepsilon}^{\boldsymbol{F}}}: \dot{\boldsymbol{\varepsilon}}^{\boldsymbol{F}}+A_{\xi^{F}} \dot{\xi}^{F} \geq 0 \\
\gamma^{R}=\boldsymbol{A}_{\boldsymbol{\varepsilon}^{R}}: \dot{\boldsymbol{\varepsilon}}^{\boldsymbol{R}}+A_{\xi^{R}} \dot{\xi}^{R} \geq 0 \\
\gamma^{r e}=\boldsymbol{A}_{\boldsymbol{\varepsilon}^{r \boldsymbol{e}}}: \dot{\boldsymbol{\varepsilon}}^{\text {re }}+\boldsymbol{A}_{\boldsymbol{v}^{r e}}: \dot{\boldsymbol{v}}^{\boldsymbol{r e}} \geq 0
\end{array}\right.
$$

255 with $\gamma^{m}$ being the part of the dissipation induced by the mechanism denoted $\mathrm{m}$.

A thermoelastic domain $\Gamma$ is considered to exist within the coordinates of the free energy potential, in which the dissipation is zero. A hypersurface $\partial \Gamma$ is the boundary of the thermoelastic domain. This hypersurface is defined as the set of solutions of the equation:

$$
\Phi(\boldsymbol{A})=0
$$


where $\boldsymbol{A}$ is the set of all GTFs. For all given coordinates, the following inequality is satisfied:

$$
\Phi(\boldsymbol{A}) \leq 0
$$

This thermoelastic domain is described by three surfaces corresponding to the three strain mechanisms:

$$
\left\{\begin{array}{l}
\Phi^{F}\left(A_{\xi}^{F}, \boldsymbol{A}_{\boldsymbol{\varepsilon}^{F}}\right)=0 \\
\Phi^{R}\left(A_{\xi}^{R}, \boldsymbol{A}_{\boldsymbol{\varepsilon}^{R}}\right)=0 \\
\Phi^{r e}\left(A_{\xi}^{r e}, \boldsymbol{A}_{\boldsymbol{\varepsilon}^{r e}}\right)=0
\end{array}\right.
$$

The $\Phi$ function for forward transformation is given as:

$$
\Phi^{F}=A_{\xi^{F}}+\hat{\Phi}^{F}\left(\boldsymbol{A}_{\boldsymbol{\varepsilon}^{F}}\right)-Y^{F}
$$

where

$$
\hat{\Phi}^{F}\left(\boldsymbol{A}_{\boldsymbol{\varepsilon}^{F}}\right)=\hat{\Phi}^{F}(\boldsymbol{\sigma})
$$

is the function describing the forward transformation function in the space of the stress components. The choice of this function determines which effects relative to anisotropy and asymmetry during forward transformation are taken in mind (Qidwai and Lagoudas 2000 Sedlák et al, 2012, Taillard et al 2008). In the scope of this paper, a modified Prager function is chosen. It accounts for tension-compression asymmetry but not anisotropy. In a superelastic loading at constant temperature, it predicts that the SMA behavior for forward transformation depends on the stress tensor invariants and asymmetry-related parameters. Thus, it is assumed that:

$$
\hat{\Phi}^{F}(\boldsymbol{\sigma})=\sqrt{J_{2}(\boldsymbol{\sigma})}\left[1+b \frac{J_{3}(\boldsymbol{\sigma})}{J_{2}^{3 / 2}(\boldsymbol{\sigma})}\right]^{\frac{1}{n}}-k_{\sigma}
$$

For a second-order tensor $\boldsymbol{u}$, the notations $J_{2}(\boldsymbol{u})$ and $J_{3}(\boldsymbol{u})$ give the second and third invariants of its deviatoric part $\boldsymbol{u}^{\prime}$. It is reminded that they are given as:

$$
\begin{aligned}
& J_{2}(\boldsymbol{u})=\frac{1}{2} u_{i j}^{\prime} u_{i j}^{\prime} \text { and } \\
& J_{3}(\boldsymbol{u})=\frac{1}{3} u_{i j}^{\prime} u_{j k}^{\prime} u_{k i}^{\prime}
\end{aligned}
$$

using the Einstein summation for double indices. $b$ and $n$ are parameters associated with the ratio between transformation stresses in tension and compression loading. Convexity is ensured under specific conditions (Chatziathanasiou et al, 2015).

The threshold for forward transformation is given as:

$$
Y^{F}=Y_{o}^{F}+D \boldsymbol{\sigma}: \boldsymbol{\Lambda}_{\varepsilon}^{\boldsymbol{F}}-\frac{\partial \lambda^{r e}}{\partial \xi} \boldsymbol{X}: \boldsymbol{v}^{r e}
$$

The variables $D$ and $Y_{o}^{F}$ are considered material constants. They are not considered ${ }_{260}$ independent, but are calculated with the help of other material constants, see (Hartl et al, 2010). 
Likewise, the $\Phi$ function for reverse transformation:

$$
\Phi^{R}=A_{\xi^{R}}+\hat{\Phi}^{R}\left(\boldsymbol{A}_{\varepsilon^{R}}\right)-Y^{R}
$$

where

$$
\hat{\Phi}^{R}\left(\boldsymbol{A}_{\boldsymbol{\varepsilon}^{R}}\right)=\hat{\Phi}^{R}(\boldsymbol{\sigma})=-\boldsymbol{\sigma}: \overline{\boldsymbol{\varepsilon}}^{\boldsymbol{T}}
$$

and

$$
Y^{R}=Y_{o}^{R}-D \boldsymbol{\sigma}: \bar{\varepsilon}^{\boldsymbol{T}}-\frac{\partial \lambda^{r e}}{\partial \xi} \boldsymbol{X}: \boldsymbol{v}^{r \boldsymbol{e}}
$$

with $Y_{o}^{R}$ another material constant. In this work, it is taken equal to $Y_{o}^{F}$. The functions for forward and reverse transformation account for isotropic hardnening, holding as hardening parameter the martensitic fraction. On the contrary, the function for reorientation is designed to predict kinematic hardening:

$$
\Phi^{r e}=\hat{\Phi}^{r e}\left(\boldsymbol{A}_{\varepsilon^{r e}}+\boldsymbol{A}_{\boldsymbol{v}^{r e}}\right)-Y^{r e}
$$

The choice for the yield function of reorientation is also the modified Prager criterion, in order to account for tension-compression asymmetry during detwinning. Only this time taking in mind the sum of the thermodynamic forces for $\varepsilon^{r e}$ and $v^{r e}$ :

$$
\hat{\Phi}^{r e}\left(\boldsymbol{A}_{\varepsilon^{r e}}+\boldsymbol{A}_{\boldsymbol{v}^{r e}}\right)=\left|\boldsymbol{A}_{\varepsilon^{r e}}+\boldsymbol{A}_{\boldsymbol{v}^{r e}}\right|=\sqrt{J_{2}(\boldsymbol{\Sigma})}\left[1+b \frac{J_{3}(\boldsymbol{\Sigma})}{J_{2}^{3 / 2}(\boldsymbol{\Sigma})}\right]^{\frac{1}{n}}
$$

Here, the inclusive variable $\boldsymbol{\Sigma}$ is introduced as:

$$
\boldsymbol{\Sigma}=\boldsymbol{\sigma}-\left(1+\lambda^{r e}\right) \boldsymbol{X}
$$

Finally, the reorientation threshold is considered as a material constant:

$$
Y^{r e}=Y_{o}^{r e}
$$

\subsection{Evolution laws}

The activation of the evolution of the model variables depends on the satisfaction of the mechanism criteria. This is given by the Kuhn-Tucker loading conditions (Qidwai 265 and Lagoudas, 2000):

It is aimed to link the evolution of the model variables within linear relation with the rate variables given in subsection 2.2. The forward transformation tensor is expressed as:

$$
\boldsymbol{\Lambda}_{\varepsilon}^{\boldsymbol{F}}=H^{c u r} \boldsymbol{\eta}_{\boldsymbol{\sigma}}
$$

where $\boldsymbol{\eta}_{\boldsymbol{u}}$ for a second-order tensor $\boldsymbol{u}$ is given as:

$\boldsymbol{\eta}_{\boldsymbol{u}}=\left(1+b \frac{J_{3}(\boldsymbol{u})}{J_{2}^{3 / 2}(\boldsymbol{u})}\right)^{\frac{1}{n}-1}\left[\frac{\boldsymbol{u}^{\prime}}{2 \sqrt{J_{2}(\boldsymbol{u})}}+\frac{b}{6 n J_{2}^{2}(\boldsymbol{u})}\left(6 J_{2}(\boldsymbol{u}) \boldsymbol{u}^{\prime} \cdot \boldsymbol{u}^{\prime}-4 J_{2}^{2}(\boldsymbol{u}) \boldsymbol{I}+(3 n-9) J_{3}(\boldsymbol{u}) \boldsymbol{u}^{\prime}\right)\right]$

This form is chosen to accommodate experimental data demonstrating lower transformation strains under compression than under tension (Bouvet et al, 2004, Grolleau et al 2011. Chemisky et al, 2015). $H^{\text {cur }}$ is considered the same as in (Hartl et al, 2010). 
The reorientation tensor introduced in 16 is:

$$
\Lambda_{\varepsilon}^{r e}=\eta_{\Sigma}
$$

With those definitions, the rates for the partition of transformation strains is now complete:

$$
\left\{\begin{array}{l}
\dot{\varepsilon}^{\boldsymbol{F}}=\dot{\xi}^{F} \boldsymbol{\Lambda}_{\varepsilon}^{\boldsymbol{F}}=\dot{\xi}^{F} H^{c u r} \boldsymbol{\eta}_{\boldsymbol{\sigma}} \\
\dot{\varepsilon}^{\boldsymbol{F}}=\dot{\xi}^{R} \boldsymbol{\Lambda}_{\boldsymbol{\varepsilon}}^{\boldsymbol{R}}=-\dot{\xi}^{R} \bar{\varepsilon}^{\boldsymbol{T}} \\
\dot{\boldsymbol{\varepsilon}}^{r e}=\dot{p}^{r e} \boldsymbol{\Lambda}_{\boldsymbol{\varepsilon}}^{r e}=\dot{p}^{r e} \boldsymbol{\eta}_{\boldsymbol{\Sigma}}
\end{array}\right.
$$

It is noted that the direction of $\dot{\varepsilon}^{r e}$ is normal to the respective yield surface:

$$
\boldsymbol{\eta}_{\boldsymbol{\Sigma}}=\frac{\partial \Phi^{r e}}{\partial \mathbf{A}_{\boldsymbol{\varepsilon}^{r e}}}=\frac{\partial \Phi^{r e}}{\partial \boldsymbol{\sigma}}
$$

The evolution of the internal variable $\boldsymbol{v}^{r e}$ is also given normal to the reorientation yield surface:

with

$$
\dot{\boldsymbol{v}}^{r e}=\dot{p}^{r e} \boldsymbol{\Lambda}_{\boldsymbol{v}}^{r e}
$$

$$
\boldsymbol{\Lambda}_{\boldsymbol{v}}^{r e}=\frac{\partial \Phi^{r e}}{\partial \boldsymbol{A}_{\boldsymbol{v}^{r e}}}=\frac{\partial \Phi^{r e}}{\partial\left(-\left(1+\lambda^{r e}\right) \boldsymbol{X}\right)}=\boldsymbol{\eta}_{\boldsymbol{\Sigma}}
$$

As for the evolution of backstress, it is decomposed as well in the contributions of the three strain mechanisms:

$$
\dot{X}=\dot{X}^{F}+\dot{X}^{R}+\dot{X}^{r e}
$$

The parts of backstress linked to forward and reverse transformation evolve since it is assumed that all processes that eventually lead to a change of transformation strain affects the reorientation behavior. The part linked to reorientation itself is in linear relation with the respective strain part so as to allow for kinematic hardening:

$$
\left\{\begin{array}{l}
\dot{\boldsymbol{X}}^{\boldsymbol{F}}=\frac{3}{2} \dot{\xi}^{F} \varepsilon_{\max }^{r e} \boldsymbol{H}^{r e}: \frac{\boldsymbol{\sigma}^{\prime}}{|\boldsymbol{\sigma}|} \\
\dot{\boldsymbol{X}}^{\boldsymbol{R}}=-\frac{\boldsymbol{X}}{\xi} \\
\dot{\boldsymbol{X}}^{r e}=\dot{p}^{r e} \boldsymbol{H}^{r e}: \boldsymbol{\Lambda}_{\varepsilon}^{r e}
\end{array}\right.
$$

The cofactor $\boldsymbol{H}^{r e}$ is a fourth order tensor considered a material constant. In the scope of this work, it is given simply by:

$$
\boldsymbol{H}^{r e}=H^{r e} \boldsymbol{I}^{(4)}
$$

with $\boldsymbol{I}^{(4)}$ the fourth order symmetric identity tensor. It has the property:

$$
I^{(4)}: x=x
$$

for any arbitrary symmetric second-order tensor $\boldsymbol{x}$.

$\varepsilon_{\max }^{r e}$ is the maximum inelastic strain allowed to develop during martensite detwinning, usually considered less than the strain caused by phase transformation. $H^{r e}$ is a parameter controlling the hardening during reorientation. The stress allowed between start and saturation of detwinning in a uniaxial case is:

$$
\Delta \sigma^{r e o}=H^{r e} \varepsilon_{\max }^{r e}
$$


It is apparent that the variable characteristic of backstress affects only the activation of reorientation. The concurrent evolution of backstress with forward and reverse transformation, as implied by (58), is introduced to comply with the need to impose a limit for reorientation. Relevant experiments (Lagoudas, 2008) show that, just like transformation, the process of reorientation is limited: after the saturation of detwinning, the elastic part of detwinned martensite is reached. To this aim, when a threshold in the magnitude of backstress is reached, the lagrange multiplier $\lambda^{r e}$ is activated, which leads to an infinitessimal value of $\dot{p}^{r e}$ and subsequently to the depending rates of reorientation variables.

The lagrange multiplier is designed as a function of a one-dimensional argument. When it attains values close to 1 , the function is activated. For values of the argument between 0 and 1 , the function returns 0 . The exact form of the function can be found in Appendix A. The argument taken in mind in the case of reorientation is:

$$
f^{r e}=\frac{|\boldsymbol{X}|}{\xi X_{\max }}
$$

and thus the respective lagrange multiplier is:

$$
\lambda^{r e}=\lambda\left(f^{r e}\right)
$$

Here, $X_{\max }$ represents a maximum magnitude of backstress when the whole RVE is composed by martensite. According to these assumptions, the maximum magnitude of backstress at any given state is proportional to the MVF. When the value $\xi X_{\max }$ is reached, the lagrange multiplier is activated and forces the variables linked to reorientation to stop evolving, by imposing:

$$
\dot{p}^{r e}=0
$$

Accordingly, forward and reverse transformation are limited through their respective lagrange multipliers. The following functions:

$$
\lambda^{F}=\lambda(\xi)
$$

and

$$
\lambda^{R}=\lambda(1-\xi)
$$

are added into the forward and reverse transformation criteria respectively. When activated, they mark the saturation of transformation by imposing $\dot{\xi}^{F}=0$ or $\dot{\xi}^{R}=0$. The criteria 42 and 45 are updated:

$$
\begin{gathered}
\Phi^{F}=A_{\xi^{F}}+\hat{\Phi}^{F}\left(\boldsymbol{A}_{\boldsymbol{\varepsilon}^{F}}\right)+\lambda^{F}-Y^{F} \\
\Phi^{R}=A_{\xi^{R}}+\hat{\Phi}^{R}\left(\boldsymbol{A}_{\boldsymbol{\varepsilon}^{R}}\right)+\lambda^{R}-Y^{R}
\end{gathered}
$$

At this point, to clear the overall view of the reader, a summarizing table providing the general architecture of the model is presented. In Table 1, the most important equations are classified by the mechanism that they concern. 


\begin{tabular}{|c|c|c|}
\hline Forward transformation & Reverse transformation & Reorientation \\
\hline $\begin{array}{c}\hat{\Phi}^{F}(\boldsymbol{\sigma})+A_{\xi^{F}}-Y^{F}+\lambda^{F} \leq 0 \\
\dot{\boldsymbol{\varepsilon}}^{\boldsymbol{F}}=\dot{\xi}^{F} \boldsymbol{\Lambda}_{\varepsilon}^{\boldsymbol{F}} \\
\dot{\boldsymbol{X}}^{\boldsymbol{F}}=\dot{\xi}^{F} \boldsymbol{\Lambda}_{\boldsymbol{X}}^{\boldsymbol{F}}\end{array}$ & $\begin{array}{c}\hat{\Phi}^{R}\left(\boldsymbol{\sigma}, \varepsilon^{\boldsymbol{T}}\right)+A_{\xi^{R}}-Y^{R}+\lambda^{R} \leq 0 \\
\dot{\varepsilon}^{\boldsymbol{R}}=\dot{\xi}^{R} \boldsymbol{\Lambda}_{\varepsilon}^{\boldsymbol{R}} \\
\dot{\varepsilon}^{\boldsymbol{R}}=\dot{\xi}^{R} \boldsymbol{\Lambda}_{\varepsilon}^{\boldsymbol{R}}\end{array}$ & $\begin{array}{c}\hat{\Phi}^{r e}(\boldsymbol{\Sigma})-Y^{r e} \leq 0 \\
\dot{\varepsilon}^{r e}=\dot{p}^{r e} \boldsymbol{\Lambda}_{\varepsilon}^{r e} \\
\dot{\boldsymbol{X}}^{r e}=\dot{p}^{r e} \boldsymbol{\Lambda}_{\boldsymbol{X}}^{r e} \\
\boldsymbol{\Sigma}=\boldsymbol{\sigma}-\left(1+\lambda^{r e}\right) \boldsymbol{X}\end{array}$ \\
\hline \multicolumn{3}{|l|}{ Composition } \\
\hline $\begin{array}{l}\xi=\xi^{F}-\xi^{R} \\
\varepsilon^{\boldsymbol{T}}=\varepsilon^{\boldsymbol{F}}+\varepsilon^{\boldsymbol{R}}+\varepsilon^{r \boldsymbol{e}} \\
\varepsilon=\varepsilon^{e l}+\varepsilon^{t \boldsymbol{h}}+\boldsymbol{\varepsilon}^{\boldsymbol{T}} \\
\varepsilon^{t \boldsymbol{h}}=\boldsymbol{\alpha}(\xi) \Delta T \\
\varepsilon^{e l}=S(\xi): \boldsymbol{\sigma} \\
\boldsymbol{X}=\boldsymbol{X}^{\boldsymbol{F}}+\boldsymbol{X}^{\boldsymbol{R}}+\boldsymbol{X}^{\boldsymbol{r e}}\end{array}$ & & \\
\hline
\end{tabular}

Table 1: Most important relations of the proposed model.

\section{Numerical implementation}

In the scope of this section, it is aimed to provide a framework for addressing the numerical implementation of a generic model that considers multiple strain mechanisms. Initially, a general solution taking in mind a random number of strain mechanisms will

285 be examined. Next, the framework will be reduced to the specific needs of the proposed SMA model. It is important to note that the added value of such general description, contrary to an ad-hoc algorithm, will allow to include additional mechanisms, for instance viscoplasticity in the case of High Temperature SMA (HTSMAs) (Chatzigeorgiou and Lagoudas, 2009, Chemisky et al, 2014), or plasticity (Hartl and Lagoudas, 2008).

290 4.1. Implicit formulation for fully coupled problem

The evolution of inelastic strains is due to several mechanisms which are considered strongly thermodynamically independent. This means that the dissipation caused by each one of them must be non-negative. The total inelastic strain is:

$$
\varepsilon^{T}=\sum_{m} \varepsilon^{m}
$$

where the index $m$ stands for every different mechanism. Each mechanism $m$ is responsible for the evolution of $\varepsilon^{m}$.

Considering each $\varepsilon^{m}$ as an internal variable, the GTF for each one deriving from the Gibbs free energy potential is:

$$
-\frac{\partial G}{\partial \varepsilon^{m}}=\sigma-\frac{\partial G^{v}}{\partial \varepsilon^{m}}
$$

where $G^{v}$ is found in the general form of $G$ :

$$
G=-\sigma: \varepsilon^{T}+G^{v}
$$


Each mechanism $\mathrm{m}$ involves a set of variables $\left\{\boldsymbol{V}_{\boldsymbol{i}}^{\boldsymbol{m}}\right\}$. Each different $\boldsymbol{V}_{\boldsymbol{i}}^{\boldsymbol{m}}$ might be considered an internal variable or not. The first element of every set is defined to be a scalar $p^{m}$ :

$$
p^{m}=V_{1}^{m}
$$

. The property of every $p^{j}$ is that the rate of all other variables $\boldsymbol{V}_{\boldsymbol{i}}^{\boldsymbol{j}}$ are found by:

$$
\dot{\boldsymbol{V}}_{i}^{j}=\dot{p}^{j} \boldsymbol{\Lambda}_{i}^{j}
$$

with $\mathrm{j}$ being an index denoting any mechanism $\mathrm{m}$ consistently throughout this section. $\boldsymbol{\Lambda}_{\boldsymbol{i}}^{j}$ is a tensor of order which varies according to the nature of $\boldsymbol{V}_{\boldsymbol{i}}^{\boldsymbol{j}}$. In the scope of this section, they are given the name evolution tensors. For instance,

$\Lambda_{1}^{j}=\Lambda_{p}^{j}=1$ is a zero order tensor equal to the unit so as to comply with 70 . Likewise,

$\Lambda_{2}^{j}=\Lambda_{\varepsilon}^{j}$ is a second-order tensor corresponding to $\varepsilon^{j}$. The second element of every set $\left\{\boldsymbol{V}_{\boldsymbol{i}}^{\boldsymbol{j}}\right\}$ will consistently be considered $\varepsilon^{j}$. GTF:

In thermodynamics, any element $\boldsymbol{V}_{\boldsymbol{i}}^{\boldsymbol{j}}$ which is an internal variable, has a conjugant

$$
\boldsymbol{A}_{\boldsymbol{V} i}^{\boldsymbol{m}}=-\frac{\partial G}{\partial \boldsymbol{V}_{\boldsymbol{i}}^{\boldsymbol{m}}}
$$

The thermodynamic criteria which govern the activation of each mechanism have the general form:

$$
\Phi^{m}\left(\left\{\boldsymbol{A}_{\boldsymbol{V}}^{m}\right\}\right) \leq 0
$$

30 where $\left\{A_{V}^{m}\right\}$ is the whole set of GTFs for the mechanism m.

For a given loading case, the convex cutting plane (CCP) (Hartl and Lagoudas, 2009) algorithm is implemented. For every iteration $\mathrm{k}$ within a loading step $n \rightarrow n+1$, it is considered that:

$$
\boldsymbol{\delta} \boldsymbol{V}_{\boldsymbol{i} n+1}^{\boldsymbol{m}(k)}=\delta p_{n+1}^{m(k)} \boldsymbol{\Lambda}_{\boldsymbol{i} n+1}^{\boldsymbol{m}(k)}
$$

The symbol $\delta$ denotes the difference in a value of a variable between two consecutive iterations in the CCP scheme.

During iterative correction, the total current strain and temperature are held constant such that:

$$
\boldsymbol{\delta} \boldsymbol{\varepsilon}_{n+1}^{(k)}=\mathbf{0}
$$

and

$$
\delta T_{n+1}^{(k)}=0
$$

According to the decomposition of strains:

$$
\varepsilon=\varepsilon^{e l}+\varepsilon^{t h}+\varepsilon^{T}=\varepsilon^{e l}+\varepsilon^{t h}+\sum_{j} \varepsilon^{j}
$$

the constitutive relation for elasticity:

$$
\varepsilon^{e l}=S: \sigma
$$

and the null increments of $\varepsilon_{n+1}$ and $T_{n+1}$, it is deduced that:

$$
-\delta \boldsymbol{\varepsilon}_{n+1}^{\boldsymbol{t h}(k)}-\sum_{j}\left(\boldsymbol{\delta} \boldsymbol{\varepsilon}_{n+1}^{\boldsymbol{j}(k)}\right)=\boldsymbol{\delta} \boldsymbol{S}_{n+1}^{(k)}: \boldsymbol{\sigma}_{n+1}^{(k)}+\boldsymbol{S}_{n+1}^{(k)}: \boldsymbol{\delta} \boldsymbol{\sigma}_{n+1}^{(k)}
$$


According to (73),

$$
\boldsymbol{\delta}_{n+1}^{\boldsymbol{j}(k)}=\delta p_{n+1}^{j(k)} \boldsymbol{\Lambda}_{\boldsymbol{\varepsilon} n+1}^{\boldsymbol{j}(k)}
$$

If the elastic compliance tensor and the thermal expansion coefficient are considered a direct function of the variables $p^{j}$, it also evident that:

$$
\boldsymbol{\delta} \boldsymbol{S}_{n+1}^{(k)}=\sum_{j}\left(\delta p_{n+1}^{j(k)} \frac{\partial \boldsymbol{S}_{n+1}^{(k)}}{\partial p^{j}}\right)
$$

and

$$
\boldsymbol{\delta} \boldsymbol{\alpha}_{n+1}^{(k)}=\sum_{j}\left(\delta p_{n+1}^{j(k)} \frac{\partial \boldsymbol{\alpha}_{n+1}^{(k)}}{\partial p^{j}}\right)
$$

With the help of $(77),(78)$ and $(79)$, the equation $(76)$ is now written:

$$
\begin{gathered}
-\Delta T_{n+1} \sum_{j}\left(\delta p_{n+1}^{j(k)} \frac{\partial \boldsymbol{\alpha}_{n+1}^{(k)}}{\partial p^{j}}\right)-\sum_{j}\left(\delta p_{n+1}^{j(k)} \boldsymbol{\Lambda}_{\boldsymbol{\varepsilon} n+1}^{j(k)}\right)=\sum_{j}\left(\delta p_{n+1}^{j(k)} \frac{\partial \boldsymbol{S}_{n+1}^{(k)}}{\partial p^{j}}\right): \boldsymbol{\sigma}_{n+1}^{(k)}+\boldsymbol{S}_{n+1}^{(k)}: \delta \boldsymbol{\sigma}_{n+1}^{(k)} \\
\Leftrightarrow \boldsymbol{\delta} \boldsymbol{\sigma}_{n+1}^{(k)}=-\boldsymbol{C}_{n+1}^{(k)}: \sum_{j}\left[\delta p_{n+1}^{j(k)}\left(\frac{\partial \boldsymbol{S}_{n+1}^{(k)}}{\partial p^{j}}: \boldsymbol{\sigma}_{n+1}^{(k)}+\Delta T_{n+1} \frac{\partial \boldsymbol{\alpha}_{n+1}^{(k)}}{\partial p^{j}}+\boldsymbol{\Lambda}_{\boldsymbol{\varepsilon} n+1}^{j(k)}\right)\right]
\end{gathered}
$$

The following notation is accepted:

$$
\boldsymbol{K}_{n+1}^{\boldsymbol{j}(k)}=\frac{\partial \boldsymbol{S}_{n+1}^{(k)}}{\partial p^{j}}: \boldsymbol{\sigma}_{n+1}^{(k)}+\Delta T_{n+1} \frac{\partial \boldsymbol{\alpha}_{n+1}^{(k)}}{\partial p^{j}}+\boldsymbol{\Lambda}_{\boldsymbol{\varepsilon} n+1}^{\boldsymbol{j}(k)}
$$

to reach:

$$
\boldsymbol{\delta} \boldsymbol{\sigma}_{n+1}^{(k)}=-\boldsymbol{C}_{n+1}^{(k)}: \sum_{j}\left(\delta p_{n+1}^{j(k)} \boldsymbol{K}_{n+1}^{j(k)}\right)
$$

On the other hand, the consistency condition of the $\Phi^{m}$ criteria dictates that:

$$
\Phi_{n+1}^{m(k)}+\delta \Phi_{n+1}^{m(k)}=\Phi_{n+1}^{m(k+1)} \approx 0
$$

From (72), in decomposing $\boldsymbol{A}_{\boldsymbol{V}}^{\boldsymbol{m}}$, the following general form is reached:

$$
\Phi^{m}\left(\boldsymbol{\sigma}, T,\left\{\boldsymbol{V}_{\boldsymbol{i}}^{\boldsymbol{j}}\right\}\right) \leq 0
$$

In the latter, applying the chain rule it is derived:

$$
\delta \Phi_{n+1}^{m(k)}=\frac{\partial \Phi_{n+1}^{m(k)}}{\partial \boldsymbol{\sigma}}: \boldsymbol{\delta} \boldsymbol{\sigma}_{n+1}^{(k)}+\sum_{j}\left[\sum_{i}\left(\frac{\partial \Phi_{n+1}^{m(k)}}{\partial \boldsymbol{V}_{\boldsymbol{i}}^{\boldsymbol{j}}}: \boldsymbol{\delta} \boldsymbol{V}_{\boldsymbol{i}(k+1}^{\boldsymbol{j}(k)}\right)\right]
$$

In light of 80 , this is written:

$$
\delta \Phi_{n+1}^{m(k)}=-\frac{\partial \Phi_{n+1}^{m(k)}}{\partial \boldsymbol{\sigma}}: \boldsymbol{C}_{n+1}^{(k)}: \sum_{j}\left(\delta p_{n+1}^{j(k)} \boldsymbol{K}_{n+1}^{\boldsymbol{j}(k)}\right)+\sum_{j}\left[\delta p_{n+1}^{j(k)} \sum_{i}\left(\frac{\partial \Phi_{n+1}^{m(k)}}{\partial \boldsymbol{V}_{\boldsymbol{i}}^{\boldsymbol{j}}}: \boldsymbol{\Lambda}_{\boldsymbol{i}}^{\boldsymbol{j}(k)}\right)\right] \Leftrightarrow
$$




$$
\delta \Phi_{n+1}^{m(k)}=\sum_{j}\left\{\delta p_{n+1}^{j(k)}\left[-\frac{\partial \Phi_{n+1}^{m(k)}}{\partial \boldsymbol{\sigma}}: \boldsymbol{C}_{n+1}^{(k)}: \boldsymbol{K}_{n+1}^{\boldsymbol{j}(k)}+\sum_{i}\left(\frac{\partial \Phi_{n+1}^{m(k)}}{\partial \boldsymbol{V}_{\boldsymbol{i}}^{\boldsymbol{j}}}: \boldsymbol{\Lambda}_{\boldsymbol{i}}^{\boldsymbol{j}(k)}\right)\right]\right\}
$$

The equation 81 will be:

$$
\delta \Phi_{n+1}^{m(k)}=\sum_{j}\left\{\delta p_{n+1}^{j(k)}\left[-\frac{\partial \Phi_{n+1}^{m(k)}}{\partial \boldsymbol{\sigma}}: \boldsymbol{C}_{n+1}^{(k)}: \boldsymbol{K}_{n+1}^{\boldsymbol{j}(k)}+\sum_{i}\left(\frac{\partial \Phi_{n+1}^{m(k)}}{\partial \boldsymbol{V}_{\boldsymbol{i}}^{\boldsymbol{j}}}: \boldsymbol{\Lambda}_{\boldsymbol{i}}^{\boldsymbol{j}(k)}\right)\right]\right\}=-\Phi_{n+1}^{m(k)}
$$

Each of the equations out of the set of (85) corresponds to each of the mechanisms $\mathrm{m}$. There are as many equations in (85) as the total number of $\mathrm{m}\left(N_{m}\right)$ and therefore the total number of $p^{m}$. A set of linear equations of $\left\{\delta p_{n+1}^{j(k)}\right\}$ is recognized in the form of:

$$
\boldsymbol{B}_{n+1}^{(k)}\left\{\delta p_{n+1}^{j(k)}\right\}=-\left\{\Phi_{n+1}^{m(k)}\right\}
$$

$\boldsymbol{B}_{n+1}^{(k)}$ is a $N_{m} \times N_{m}$ matrix, the components of which are found by:

$$
B_{j n+1}^{m(k)}=B_{m j}{ }_{n+1}^{(k)}=-\frac{\partial \Phi_{n+1}^{m(k)}}{\partial \boldsymbol{\sigma}}: \boldsymbol{C}_{n+1}^{(k)}: \boldsymbol{K}_{n+1}^{\boldsymbol{j}(k)}+\sum_{i}\left(\frac{\partial \Phi_{n+1}^{m(k)}}{\partial \boldsymbol{V}_{\boldsymbol{i}}^{\boldsymbol{j}}}: \boldsymbol{\Lambda}_{\boldsymbol{i}}^{\boldsymbol{j}(k)}{ }_{n+1}\right)
$$

From 86 , the set of $\delta p^{j(k)}$ is found by:

$$
\left\{\delta p_{n+1}^{j(k)}\right\}=-\left(\boldsymbol{B}_{n+1}^{(k)}\right)^{-1}\left\{\Phi_{n+1}^{m(k)}\right\}
$$

At the end of every iteration the values of the set of $\left\{\boldsymbol{V}_{\boldsymbol{i}}^{\boldsymbol{m}}\right\}$ and the rest of the internal variables.

\section{4.2. Thermomechanical tangent moduli estimation}

During an arbitrary thermomechanical path, the mechanical and thermal tangent moduli need to be calculated at the end of every loading step. Again, the additive decomposition of strains and their respective rates is taken in mind:

$$
d \varepsilon=d \varepsilon^{e l}+d \varepsilon^{t h}+\sum_{j} d \varepsilon^{j}
$$

where the sum denoted by $\sum_{j}$ refers only to the mechanisms $\mathrm{m}$ that are activated in the current step. $\mathrm{j}$ may take the values $\mathrm{F}, \mathrm{R}$ or re. Given that these moduli examine the tangent behavior between two consecutive steps, the symbol $d$ denotes the difference in the value of the variables between those steps.

The previous equation gives:

$$
\boldsymbol{d} \varepsilon=\boldsymbol{d}(\boldsymbol{S}: \boldsymbol{\sigma})+\boldsymbol{d}(\boldsymbol{\alpha} \Delta T)+\sum_{j}\left(d p^{j} \boldsymbol{\Lambda}_{\varepsilon}^{\boldsymbol{j}}\right) \Leftrightarrow
$$




$$
\boldsymbol{d} \boldsymbol{\sigma}=\boldsymbol{C}:\left[\boldsymbol{d} \boldsymbol{\varepsilon}-\sum_{j}\left(d p^{j} \boldsymbol{K}^{j}\right)-\boldsymbol{\alpha} d T\right]
$$

where

$$
\boldsymbol{K}^{j}=\frac{\partial \boldsymbol{S}}{\partial p^{j}}: \boldsymbol{\sigma}+\frac{\partial \boldsymbol{\alpha}}{\partial p^{j}} \Delta T+\boldsymbol{\Lambda}_{\boldsymbol{\varepsilon}}^{j}
$$

For all different mechanisms $\mathrm{m}$, it is also assumed: $d \Phi^{m}=0$. But:

$$
d \Phi^{m}=\frac{\partial \Phi^{m}}{\partial \boldsymbol{\sigma}}: \boldsymbol{d} \boldsymbol{\sigma}+\frac{\partial \Phi^{m}}{\partial T} d T+\sum_{j}\left[\sum_{i}\left(\frac{\partial \Phi^{m}}{\partial \boldsymbol{V}_{\boldsymbol{i}}^{\boldsymbol{j}}}: \boldsymbol{d} \boldsymbol{V}_{\boldsymbol{i}}^{\boldsymbol{j}}\right)\right]
$$

In light of $(70)$, this is written:

$$
d \Phi^{m}=\frac{\partial \Phi^{m}}{\partial \boldsymbol{\sigma}}: \boldsymbol{d} \boldsymbol{\sigma}+\frac{\partial \Phi^{m}}{\partial T} d T+\sum_{j}\left[d p^{j} \sum_{i}\left(\frac{\partial \Phi^{m}}{\partial \boldsymbol{V}_{\boldsymbol{i}}^{\boldsymbol{j}}}: \boldsymbol{\Lambda}_{\boldsymbol{i}}^{j}\right)\right]
$$

Substituting with the expression of $\boldsymbol{d} \boldsymbol{\sigma}$ from 90 , it is found:

$$
d \Phi^{m}=\frac{\partial \Phi^{m}}{\partial \boldsymbol{\sigma}}: \boldsymbol{C}:\left[\boldsymbol{d} \boldsymbol{\varepsilon}-\sum_{j}\left(d p^{j} \boldsymbol{K}^{\boldsymbol{j}}\right)-\boldsymbol{\alpha} d T\right]+\frac{\partial \Phi^{m}}{\partial T} d T+\sum_{j}\left[d p^{j} \sum_{i}\left(\frac{\partial \Phi^{m}}{\partial \boldsymbol{V}_{\boldsymbol{i}}^{j}}: \boldsymbol{\Lambda}_{\boldsymbol{i}}^{j}\right)\right]
$$

and, given the null increment of the $\Phi^{m}$ functions:

$$
\frac{\partial \Phi^{m}}{\partial \boldsymbol{\sigma}}: \boldsymbol{C}: \boldsymbol{d} \boldsymbol{\varepsilon}+\left(\frac{\partial \Phi^{m}}{\partial T}-\frac{\partial \Phi^{m}}{\partial \boldsymbol{\sigma}}: \boldsymbol{C}: \boldsymbol{\alpha}\right) d T+\sum_{j}\left\{d p^{j}\left[\sum_{i}\left(\frac{\partial \Phi^{m}}{\partial \boldsymbol{V}_{\boldsymbol{i}}^{\boldsymbol{j}}}: \boldsymbol{\Lambda}_{\boldsymbol{i}}^{j}\right)-\frac{\partial \Phi^{m}}{\partial \boldsymbol{\sigma}}: \boldsymbol{C}: \boldsymbol{K}^{j}\right]\right\}=0
$$

There are as many equations in $(94)$ as the total number of $\mathrm{m}\left(N_{m}\right)$ that are activated for the current step and therefore the total number of $p^{m}$.A set of linear equations of the vector $\left\{d p^{j}\right\}$ is recognized in the form of:

$$
\hat{\boldsymbol{B}}\left\{d p^{j}\right\}=\left\{-\frac{\partial \Phi^{m}}{\partial \boldsymbol{\sigma}}: \boldsymbol{C}: \boldsymbol{d} \boldsymbol{\varepsilon}-\left(\frac{\partial \Phi^{m}}{\partial T}-\frac{\partial \Phi^{m}}{\partial \boldsymbol{\sigma}}: \boldsymbol{C}: \boldsymbol{\alpha}\right) d T\right\}
$$

where: $\hat{\boldsymbol{B}}$ is a $N_{m} \times N_{m}$ matrix, the components $\hat{B}_{m j}$ of which are found by:

$$
\hat{B}_{m j}=\sum_{i}\left(\frac{\partial \Phi^{m}}{\partial \boldsymbol{V}_{\boldsymbol{i}}^{\boldsymbol{j}}}: \boldsymbol{\Lambda}_{\boldsymbol{i}}^{\boldsymbol{j}}\right)-\frac{\partial \Phi^{m}}{\partial \boldsymbol{\sigma}}: \boldsymbol{C}: \boldsymbol{K}^{\boldsymbol{j}}
$$

Following (95),

$$
\left\{d p^{j}\right\}=\hat{\boldsymbol{B}}^{-\mathbf{1}}\left\{-\frac{\partial \Phi^{m}}{\partial \boldsymbol{\sigma}}: \boldsymbol{C}: \boldsymbol{d} \boldsymbol{\varepsilon}-\left(\frac{\partial \Phi^{m}}{\partial T}-\frac{\partial \Phi^{m}}{\partial \boldsymbol{\sigma}}: \boldsymbol{C}: \boldsymbol{\alpha}\right) d T\right\}
$$

or

$$
d p^{j}=-\sum_{m}\left(\hat{B}_{m j}^{-1} \frac{\partial \Phi^{m}}{\partial \boldsymbol{\sigma}}: \boldsymbol{C}\right): \boldsymbol{d} \varepsilon-\sum_{m}\left[\hat{B}_{m j}^{-1}\left(\frac{\partial \Phi^{m}}{\partial T}-\frac{\partial \Phi^{m}}{\partial \boldsymbol{\sigma}}: \boldsymbol{C}: \boldsymbol{\alpha}\right)\right] d T
$$


Here, the following notations are defined:

$$
P_{T}^{j}=\sum_{m}\left[\hat{B}_{m j}^{-1}\left(\frac{\partial \Phi^{m}}{\partial T}-\frac{\partial \Phi^{m}}{\partial \boldsymbol{\sigma}}: \boldsymbol{C}: \boldsymbol{\alpha}\right)\right]
$$

and

$$
\boldsymbol{P}_{\varepsilon}^{\boldsymbol{j}}=\sum_{m}\left(\hat{B}_{m j}^{-1} \frac{\partial \Phi^{m}}{\partial \boldsymbol{\sigma}}: \boldsymbol{C}\right)
$$

Given the major symmetry $C_{u v s t}=C_{\text {stuv }}$ of the elastic stiffness tensor, the latter is now:

$$
\boldsymbol{P}_{\varepsilon}^{\boldsymbol{j}}=\boldsymbol{C}: \sum_{m}\left(\hat{B}_{m j}^{-1} \frac{\partial \Phi^{m}}{\partial \boldsymbol{\sigma}}\right)
$$

Thus, the equation (97) is now updated:

$$
d p^{j}=-\boldsymbol{P}_{\varepsilon}^{j}: \boldsymbol{d} \varepsilon-P_{T}^{j} d T
$$

Substituting this expression for $d p^{j}$ in 90 , it is deduced:

$$
\boldsymbol{d} \boldsymbol{\sigma}=\left\{\boldsymbol{C}:\left[\boldsymbol{I}^{(4)}+\sum_{j}\left(\boldsymbol{K}^{j} \otimes \boldsymbol{P}_{\varepsilon}^{j}\right)\right]\right\}: \boldsymbol{d} \boldsymbol{\varepsilon}+\left\{\boldsymbol{C}:\left[\sum_{j}\left(P_{T}^{j} \boldsymbol{K}^{j}\right)-\boldsymbol{\alpha}\right]\right\} d T
$$

Here, the desired tangent moduli become evident. The mechanical tangent modulus is:

$$
\boldsymbol{L}=\boldsymbol{C}:\left[\boldsymbol{I}^{(4)}+\sum_{j}\left(\boldsymbol{K}^{j} \otimes \boldsymbol{P}_{\varepsilon}^{j}\right)\right]
$$

and the thermal tangent modulus:

$$
\boldsymbol{\Theta}=\boldsymbol{C}:\left[\sum_{j}\left(P_{T}^{j} \boldsymbol{K}^{\boldsymbol{j}}\right)-\boldsymbol{\alpha}\right]
$$

\subsection{Thermomechanical coupling}

The local form of the first law of thermodynamics is written:

$$
\dot{u}=r-\nabla \cdot \boldsymbol{q}+\boldsymbol{\sigma}: \dot{\varepsilon}=\dot{Q}+\boldsymbol{\sigma}: \dot{\varepsilon}
$$

Here, $u$ is the internal energy, $r$ is the power of the internally generated heat, $\boldsymbol{q}$ is the thermal flux and $Q$ is the total heat exchange due to both internal heat sources and conduction. On the other hand, the Gibbs free energy is given as:

$$
G=u-\sigma: \varepsilon-s T
$$

Its rate form:

$$
\dot{u}=\dot{G}+(\sigma: \varepsilon)+(\dot{s})
$$


is replaced into 105 to give:

$$
\dot{G}+\dot{\sigma}: \varepsilon+\dot{s} T+s \dot{T}=\dot{Q}
$$

Applying the chain rule to derive the rate of the Gibbs free energy potential, it is found:

$$
\dot{G}=\frac{\partial G}{\partial \boldsymbol{\sigma}}: \dot{\boldsymbol{\sigma}}+\frac{\partial G}{\partial T} \dot{T}+\sum_{j}\left[\sum_{i}\left(\frac{\partial G}{\partial \boldsymbol{V}_{\boldsymbol{i}}^{\boldsymbol{j}}}: \dot{\boldsymbol{V}}_{\boldsymbol{i}}^{\boldsymbol{j}}\right)\right]
$$

Recalling equations (32), 33, ) 700 and (71), this rate is now:

$$
\dot{G}=-\varepsilon: \dot{\sigma}-s \dot{T}-\sum_{j}\left[\dot{p}^{j} \sum_{i}\left(\boldsymbol{A}_{i}^{j}: \Lambda_{i}^{j}\right)\right]
$$

Giving the definition:

$$
\pi^{j}=\sum_{i}\left(\boldsymbol{A}_{i}^{j}: \boldsymbol{\Lambda}_{i}^{j}\right)
$$

the equation 108 is now:

$$
\dot{s} T=\sum_{j}\left(\pi^{j} \dot{p}^{j}\right)+\dot{Q}
$$

in which $\dot{s}$ is found from 32 :

$\dot{s}=\frac{\partial s}{\partial \boldsymbol{\sigma}}: \dot{\boldsymbol{\sigma}}+\frac{\partial s}{\partial T} \dot{T}+\sum_{j}\left(\frac{\partial s}{\partial p^{j}} \dot{p}^{j}\right)=\dot{\boldsymbol{\sigma}}: \boldsymbol{\alpha}+C_{v} \frac{\dot{T}}{T}+\sum_{j}\left\{\dot{p}^{j}\left[\frac{\partial s_{o}}{\partial p^{j}}+\boldsymbol{\sigma}: \frac{\partial \boldsymbol{\alpha}}{\partial p^{j}}+\frac{\partial C_{v}}{\partial p^{j}} \ln \left(\frac{T}{T_{o}}\right)\right]\right\}$

Thus, from 112:

$$
C_{v} \dot{T}=\sum_{j}\left\{\dot{p}^{j}\left[\pi^{j}-\left[\frac{\partial s_{o}}{\partial p^{j}}+\boldsymbol{\sigma}: \frac{\partial \boldsymbol{\alpha}}{\partial p^{j}}+\frac{\partial C_{v}}{\partial p^{j}} \ln \left(\frac{T}{T_{o}}\right)\right] T\right]\right\}-\dot{\boldsymbol{\sigma}}: \boldsymbol{\alpha} \cdot T+\dot{Q}
$$

The following notation is adopted:

$$
\pi_{T}^{j}=\pi^{j}-\left[\frac{\partial s_{o}}{\partial p^{j}}+\boldsymbol{\sigma}: \frac{\partial \boldsymbol{\alpha}}{\partial p^{j}}+\frac{\partial C_{v}}{\partial p^{j}} \ln \left(\frac{T}{T_{o}}\right)\right] T
$$

The known forms of $\dot{p}^{j}$ and $\dot{\boldsymbol{\sigma}}$ from 101 and 102 are also substituted into 114 to acquire:

$$
C_{v} \dot{T}-\dot{Q}=-\left[\sum_{j}\left(\pi_{T}^{j} \boldsymbol{P}_{\varepsilon}^{j}\right)+\boldsymbol{L}: \boldsymbol{\alpha} \cdot T\right]: \dot{\boldsymbol{\varepsilon}}-\left[\sum_{j}\left(\pi_{T}^{j} P_{T}^{j}\right)+\boldsymbol{\Theta}: \boldsymbol{\alpha} \cdot T\right] \dot{T}
$$

The last equation states that the volumetric heat generation per unit time is not equal to the total heat exchange. The difference between these two quantities arises by effect of mechanical work, as it appears in the right-hand part of the equation. The part of dissipation linked to mechanical reaction of the material, such as the one found in (38), 315 is also found in this part. 
The quantities

$$
D_{, \varepsilon}=-\left[\sum_{j}\left(\pi_{T}^{j} \boldsymbol{P}_{\varepsilon}^{j}\right)+\boldsymbol{L}: \boldsymbol{\alpha} \cdot T\right]
$$

and

$$
D_{, T}=-\left[\sum_{j}\left(\pi_{T}^{j} P_{T}^{j}\right)+\boldsymbol{\Theta}: \boldsymbol{\alpha} \cdot T\right]
$$

track the linear relation of the power of the heat generated through thermomechanical coupling with the differentials of strain and temperature.

\subsection{Reduction for current model}

The scalar quantities $p^{m}$ whose rates drive the three strain mechanisms are: $\xi^{F}$ for forward, $\xi^{R}$ for reverse transformation and $p^{r e}$ for reorientation. In Table 2, the relation of the rates of the model with the driving scalar rates through their respective evolution tensors $\Lambda_{V i}^{m}$.

\begin{tabular}{c|ccc} 
Evolving variables - Strain mechanisms & FT & RT & Re \\
\hline$V_{1}^{m}$ & $\xi^{F}$ & $\xi^{R}$ & $p^{r e}$ \\
$\boldsymbol{V}_{\mathbf{2}}^{m}$ & $\boldsymbol{\varepsilon}^{\boldsymbol{F}}$ & $\boldsymbol{\varepsilon}^{\boldsymbol{R}}$ & $\boldsymbol{\varepsilon}^{r \boldsymbol{e}}$ \\
$\boldsymbol{V}_{\mathbf{3}}^{\boldsymbol{m}}$ & $\boldsymbol{X}^{\boldsymbol{F}}$ & $\boldsymbol{X}^{\boldsymbol{R}}$ & $\boldsymbol{X}^{\boldsymbol{r e}}$
\end{tabular}

Table 2: Classification of evolving model variables according to the respective mechanisms

Next, the linear relations of the elastic compliance tensor and the thermal expansion coefficient with the driving scalars are reduced to:

$$
\begin{aligned}
& \frac{\partial \boldsymbol{S}}{\partial \xi^{F}}=-\frac{\partial \boldsymbol{S}}{\partial \xi^{R}}=\tilde{\boldsymbol{S}} \quad \text { and } \quad \frac{\partial \boldsymbol{S}}{\partial p^{r e}}=\mathbf{0} \\
& \frac{\partial \boldsymbol{\alpha}}{\partial \xi^{F}}=-\frac{\partial \boldsymbol{\alpha}}{\partial \xi^{R}}=\tilde{\boldsymbol{\alpha}} \quad \text { and } \quad \frac{\partial \boldsymbol{\alpha}}{\partial p^{r e}}=\mathbf{0}
\end{aligned}
$$

The strain quantities $\boldsymbol{K}^{\boldsymbol{m}}$ are reduced to:

$$
\begin{aligned}
& \boldsymbol{K}^{\boldsymbol{F}}=\tilde{\boldsymbol{S}}: \boldsymbol{\sigma}+\tilde{\boldsymbol{\alpha}} \Delta T+\boldsymbol{\Lambda}_{\varepsilon}^{\boldsymbol{F}} \\
& \boldsymbol{K}^{\boldsymbol{R}}=-\tilde{\boldsymbol{S}}: \boldsymbol{\sigma}-\tilde{\boldsymbol{\alpha}} \Delta T+\boldsymbol{\Lambda}_{\varepsilon}^{\boldsymbol{R}} \\
& \boldsymbol{K}^{r e}=\boldsymbol{\Lambda}_{\varepsilon}^{r e}
\end{aligned}
$$

Furthermore, multiple derivatives appearing in the components of the $\boldsymbol{B}$ and $\tilde{\boldsymbol{B}}$ matrices in equations (87) and (96) take zero values, since the $\Phi^{m}$ yield equations are not

325 dependent on all the evolving variables. Table 3 presents which variables affect the three yield functions. 


\begin{tabular}{l|l|l}
$\Phi^{F}$ & $\Phi^{R}$ & $\Phi^{r e}$ \\
\hline $\boldsymbol{\sigma}, T, \xi$ & $\boldsymbol{\sigma}, T, \xi, \boldsymbol{\varepsilon}^{\boldsymbol{F}}, \boldsymbol{\varepsilon}^{\boldsymbol{R}}, \boldsymbol{\varepsilon}^{\boldsymbol{r e}}$ & $\boldsymbol{\sigma}, \xi \boldsymbol{X}^{\boldsymbol{F}}, \boldsymbol{X}^{\boldsymbol{R}}, \boldsymbol{X}^{\boldsymbol{r e}}$
\end{tabular}

Table 3: Variables affecting the yield functions

\section{Numerical results and discussion}

The thermomechanical response of the proposed model is compared to several experimentally observed complex, non-proportional thermomechanical response of SMAs, to sзо the extent of their availability.

The selected experimental database includes proportional and non-proportional loading conditions in various thermal and mechanical levels.

A first set of experiments concern the isothermal tension loading of NiTi wires under various temperatures, either above or in-between martensitic transformation temperatures Sittner et al (2009). A second set of experiments corresponds to non-proportional tension-torsion loading of SMA tube structures(Sittner et al, 1995). A last set of experiment is complex tension/torsion and tension compression on NiTi tube Grabe and Bruhns (2009). Parameter identification was carried out using a proven method reliable in identifying complex modeling parameters for SMAs using inverse identification meth-

340 ods developed by Meraghni et al (2014) and extended for heterogeneous configuration in Chemisky et al (2015).

\begin{tabular}{lllllll}
\hline$E^{A}(\mathrm{MPa})$ & $E^{M}(\mathrm{MPa})$ & $v^{A}$ & $v^{M}$ & $\alpha\left(K^{-1}\right)$ & $H_{\min }$ & $H_{\text {sat }}$ \\
40000 & 23000 & $(0.33)$ & $(0.33)$ & $\left(10^{-5}\right)$ & 0.052 & 0.052 \\
& $C^{A}(\mathrm{MPa} / \mathrm{K})$ & $C^{M}(\mathrm{MPa} / \mathrm{K})$ & $M_{f}(\mathrm{~K})$ & $M_{s}(\mathrm{~K})$ & $A_{s}(\mathrm{~K})$ & $A_{f}(\mathrm{~K})$ \\
- & 7.2 & 4.8 & 198.5 & 200 & 266 & 270 \\
$Y_{o}^{\text {reo }}(\mathrm{MPa})$ & $\varepsilon_{\max }^{r e}$ & $H^{r e}$ & $\mathrm{~b}$ & $\mathrm{n}$ & & \\
100 & 0.052 & 2000 & - & - & & \\
\hline
\end{tabular}

Table 4: Material constants for equiatomic NiTi utilized in loading simulations of experiments presented in (Sittner et al, 2009). Values that are not result of identification appear in parentheses. 


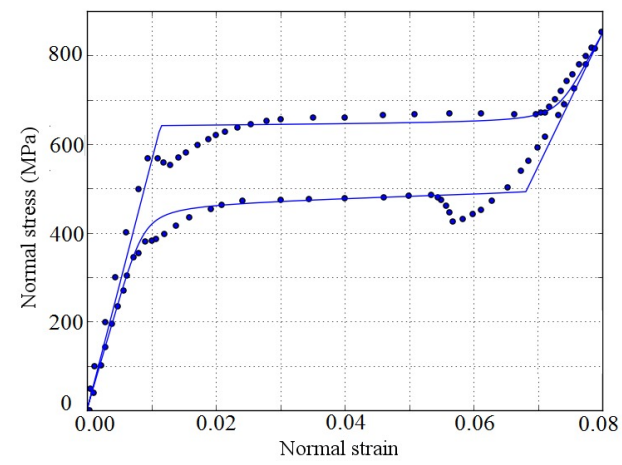

(a) Tension at $333 \mathrm{~K}$

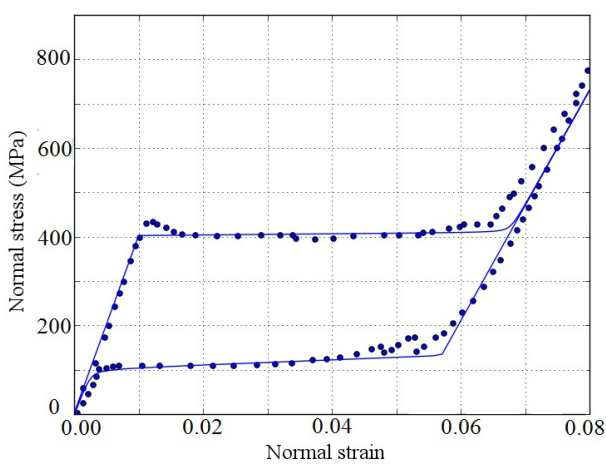

(b) Tension at $283 \mathrm{~K}$

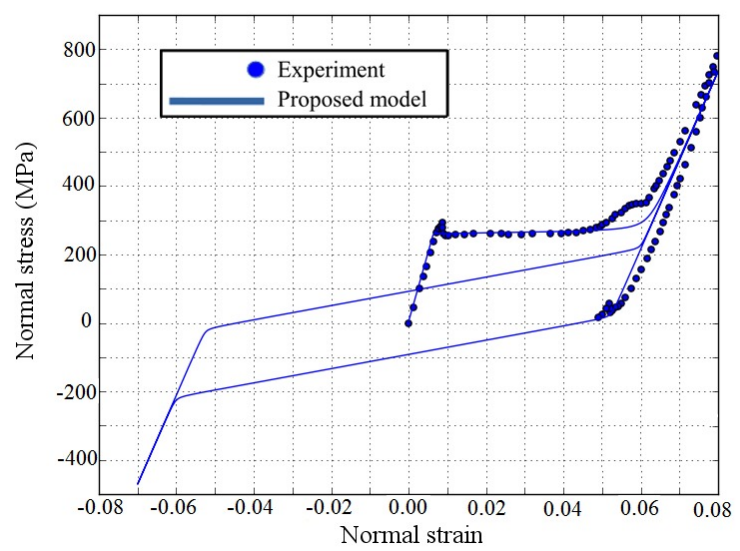

(c) Tension at $253 \mathrm{~K}$

Figure 3: Experimental results (points) and model reponse (continuous line) on stress-strain diagrams for isothermal tension-compression loading of NiTi wires under temperatures of 333 (a), 283 (b) and $253 \mathrm{~K}$ (c) taken from (Sittner et al, 2009)

\subsection{Iso-thermal proportional tension-compression loading}

Three tensile loading tests on NiTi wires of $0.1 \mathrm{~mm}$ in diameter and $50 \mathrm{~mm}$ in length are simulated in iso-thermal conditions for different temperatures The numerical model corresponds to the experiments in (Sittner et al, 2009): The displacement control of wires of $0.1 \mathrm{~mm}$ in diameter and $50 \mathrm{~mm}$ in length is taken as a strain-controlled simulation of a material point in its loading direction, and in stress-free condition in shear in other directions. In these experiments, compression cannot be carried out, since the small diameter of a wire would cause buckling immediately. In the simulations, though, inelastic geometry effects can be ignored.

For the temperature of $333 \mathrm{~K}$, the wire demonstrates a superelastic behavior, since the stress levels cross all the transition thresholds for the start and finish of forward and reverse transformation before returning to zero stress. The behavior is similar for the temperature of $283 \mathrm{~K}$, only with lower stress levels for forward and reverse transforma- 


\begin{tabular}{lllllll}
\hline$E^{A}(\mathrm{MPa})$ & $E^{M}(\mathrm{MPa})$ & $v^{A}$ & $v^{M}$ & $\alpha\left(K^{-1}\right)$ & $H_{\min }$ & $H_{\text {sat }}$ \\
38000 & 13000 & 0.20 & 0.20 & $\left(10^{-5}\right)$ & $(0.007)$ & 0.007 \\
$k$ & $C^{A}(\mathrm{MPa} / \mathrm{K})$ & $C^{M}(\mathrm{MPa} / \mathrm{K})$ & $M_{f}(\mathrm{~K})$ & $M_{s}(\mathrm{~K})$ & $A_{s}(\mathrm{~K})$ & $A_{f}(\mathrm{~K})$ \\
- & 8.7 & 7.27 & 215 & 260 & 222 & 275 \\
$Y_{o}^{\text {reo }}(\mathrm{MPa})$ & $\varepsilon_{\max }^{r e}$ & $H^{r e}$ & $\mathrm{~b}$ & $\mathrm{n}$ & & \\
398 & 0.007 & 21400 & - & - & & \\
\hline
\end{tabular}

Table 5: Material constants for CuAlZnMn tube subjected to non-proportional tension-torsion from (Sittner et al 1995). Values that are not result of identification appear in parentheses.

355 tion, following a typical SMA phase diagram. At $253 \mathrm{~K}$ though, the transformation strain during loading is induced by forward transformation activated alongside reoreintation. This strain cannot be recovered upon unloading: since the temperature is lower than the austenitic transition level, no reverse transformation can take place. Still, the proposed model can predict some lef 'vel of inelastic strain recovery just before reaching zero stress, associated with reorientation. Continuing further into compression, the material passes in a behavior affected only by reorientation. In the second cycle of loading, no transformation is considered, explaining the difference of shape from the first cycle. Good agrement is observed between the simulation and the experimental findings in tension. It is noted that the value of the elastic modulus is considered different for the test at $333 \mathrm{~K}$, taken at $56000 \mathrm{MPa}$, since the stress-strain relation clearly indicates so. The current model does not take into account any dependence of the elastic modulus from temperature. However, all other material parameters are considered constant for the three experiments. Figs. 3 (a) to (c) present the comparison of the stress-strain diagrams for the isothermal tension-compression loading paths. In Fig. 3 (c), a prediction of the material behavior in compression is also presented. Reverse transformation cannot take place at such temperature. The effect of combined transformation/reorientation leads to a transient response for the first cycle, then to a stable reorientation loop since no reverse transformation occurs. The identified parameters appear in Table 4 .

\subsection{Iso-thermal non-proportional loading}

375

Next, the proposed model is aimed at being further validated by comparing experimental results corresponding to non-proportional loading of thin-walled tube structures (Sittner et al, 1995). A cylindrical tube made of CuAlZnMn alloy was subjected to a loading path resembling a rectangle in the space of normal (axial) and shear (along the tube walls) stress, with return to zero loading. The path is represented in Fig. (4a), while

3во the material properties identified are reported in Table 5 . The model response is superimposed to experimental data points in diagrams relating shear and normal strain in Fig. (4b), normal stress and strain in (4c) and shear stress and strain in (4d). A comparison with the models proposed by Lagoudas et al (2012), Arghavani et al (2010) and Panico and Brinson (2007), who have executed the same simulation is also conducted. The

385 proposed model shows activation of the reorientation mechanism only between points $\mathrm{C}$ and $\mathrm{D}$ of the loading path, where it is combined with reverse transformation. The normal stress level reached at point $\mathrm{A}$ is low enough to allow for even more forward 

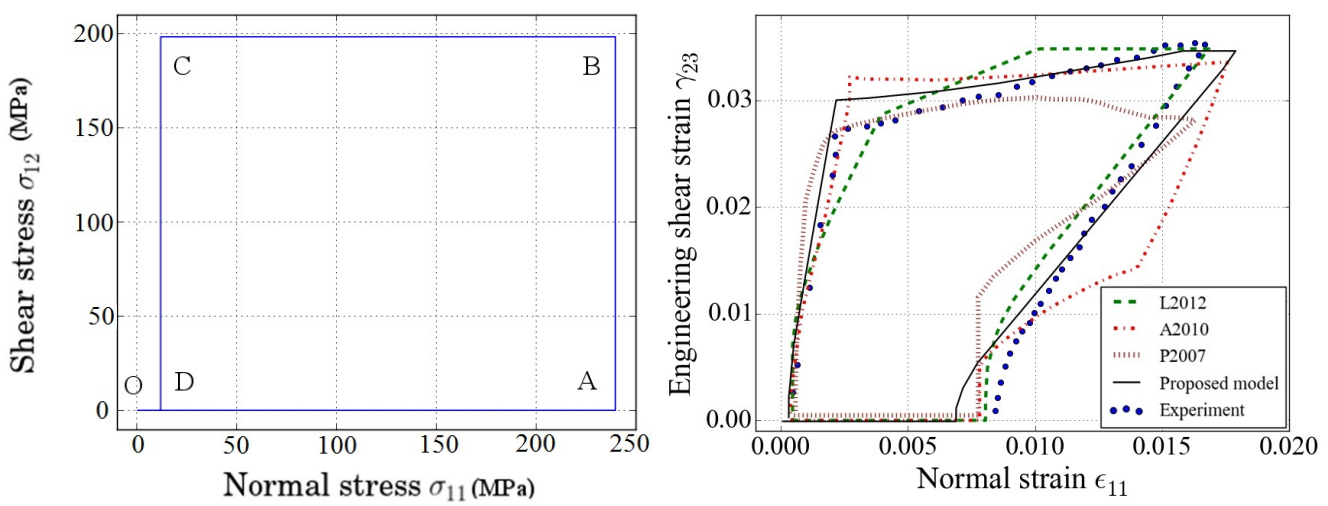

(a) Rectangle loading path in stresses on SMA (b) Material response in normal and shear tube

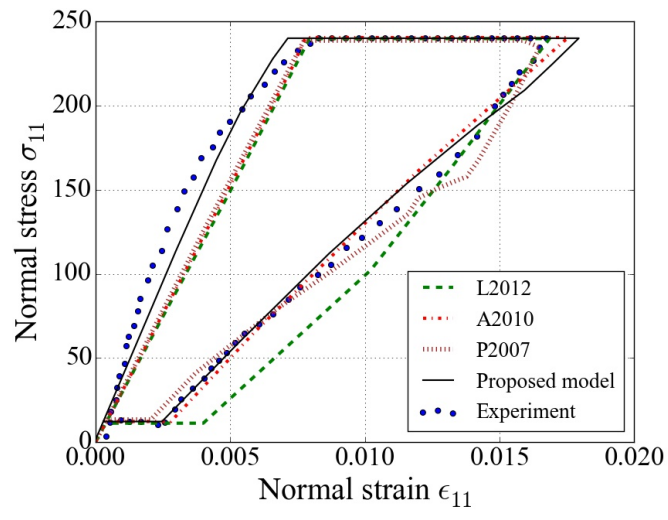

(c) Normal stress-strain diagram strains

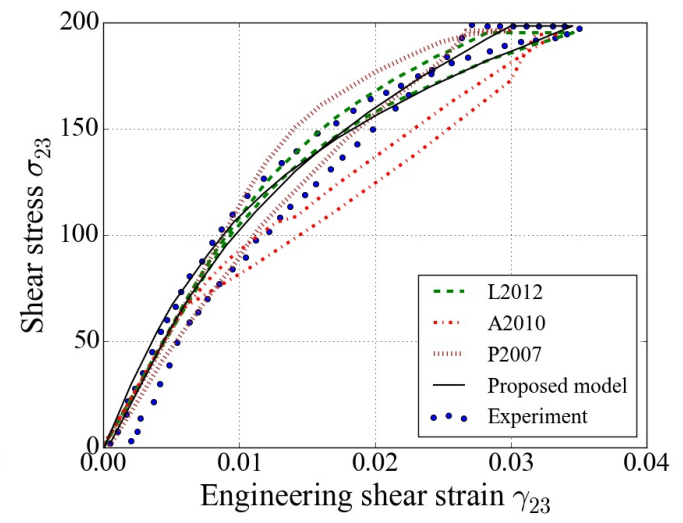

(d) Shear stress-strain diagram

Figure 4: Experimental results (points) and model reponse (continuous line) on SMA thin-walled tube from (Sittner et al, 1995). Comparison with model results from (Lagoudas et al, 2012) (L2012), (Arghavani et al 2010) (A2010) and (Panico and Brinson, 2007) (P2007).

transformation along the shear direction in the branch $\mathrm{A}$ to $\mathrm{B}$, and therefore reorientation is not activated. Sufficient accuracy of the predictive capabilities of the model in this case of non-proportional loading is demonstrated, especially in comparison with the attempts made by previous models. As a final remark, it is added that even the Young's modulus for austenite was captured with higher precision relatively to the other models, as becomes evident at the elastic phase in Fig. (4c). This improved approach to such an important material parameter demonstrates the combined adaptive capabilty of the new model and the implemented identification algorithm.

Comparing the results from simulations between the current model and the model by Lagoudas et al (2012), it becomes clear that certain aspects of material response are handled better. Specifically, after point B, reorientation is activated alongside reverse transformation when the stress levels are low enough. This has an impact on the twobranch linear response between points $\mathrm{B}$ and $\mathrm{C}$ in the shear-normal strain curve in Fig. 


\begin{tabular}{lllllll}
\hline$E^{A}(\mathrm{MPa})$ & $E^{M}(\mathrm{MPa})$ & $v^{A}$ & $v^{M}$ & $\alpha\left(K^{-1}\right)$ & $H_{\text {min }}$ & $H_{\text {sat }}$ \\
50120 & 27000 & 0.30 & 0.30 & $\left(10^{-5}\right)$ & 0.0 & 0.056 \\
$k$ & $C^{A}(\mathrm{MPa} / \mathrm{K})$ & $C^{M}(\mathrm{MPa} / \mathrm{K})$ & $M_{f}(\mathrm{~K})$ & $M_{s}(\mathrm{~K})$ & $A_{s}(\mathrm{~K})$ & $A_{f}(\mathrm{~K})$ \\
0.021 & 9 & 9 & 255 & 258 & 264 & 277 \\
$Y_{o}^{\text {reo }}(\mathrm{MPa})$ & $\varepsilon_{\text {max }}^{\text {re }}$ & $H^{\text {re }}$ & $\mathrm{b}$ & $\mathrm{n}$ & & \\
132.9 & 1546 & 0.046 & 0.608 & 2 & & \\
\hline
\end{tabular}

Table 6: Material constants for NiTi tube subjected to tension-torsion-thermal loading presented in Grabe and Bruhns 2009. Values that are not result of identification appear in parentheses.

4. (b). The transition point between the two branches is shifted to lower normal strain values, which is a direct effect of the simultaneous activation of reorientation and reverse activation. This leads to a strong reduction of normal but not shear transformation strains, following the evolution of stress. This could not have been predicted with the previous model. Moreover, in Fig. 4 (d), the crossing of the response curve in the shear stress-shear strain space is reproduced, whereas, with the previous model, the return path C-D never crosses the initial A-B curve. The difference in the predicted capacity for developing transformation strains is also evident. Whereas the previous model is presents a value of $3.5 \%$ for recoverable strain, the identification of model parameters with the current model reveals that $0.7 \%$ would be a better estimate. This implies that the non-linear effects are mostly a result of the change in elastic modulus. As the phase transformation carries through, the fading elastic modulus of martensite takes effect in

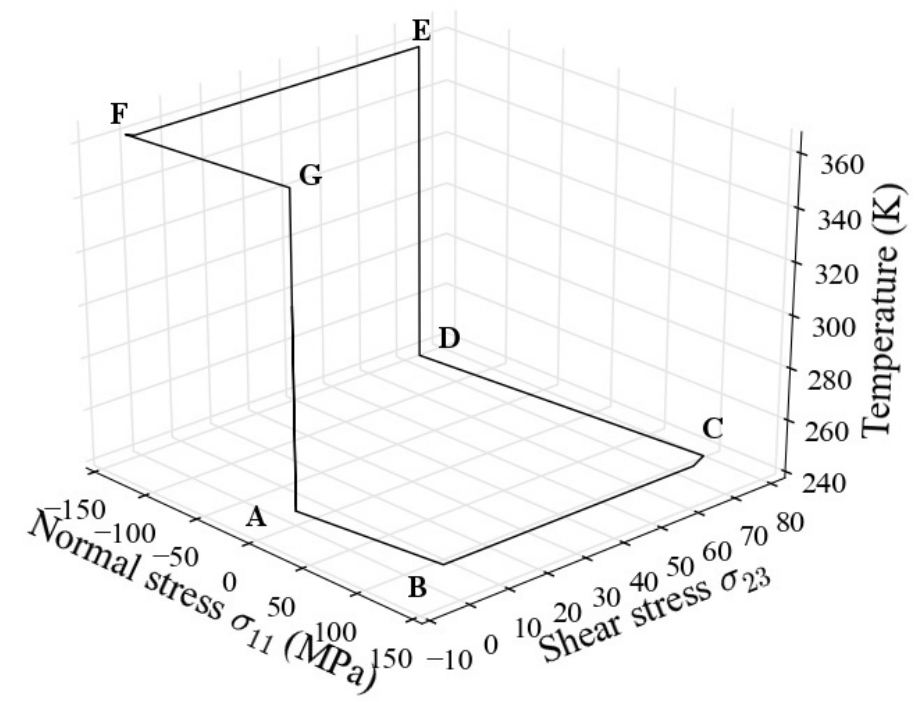

Figure 5: Tension-compression loading combined with torsion in varying temperature, taken from Grabe and Bruhns 2009 


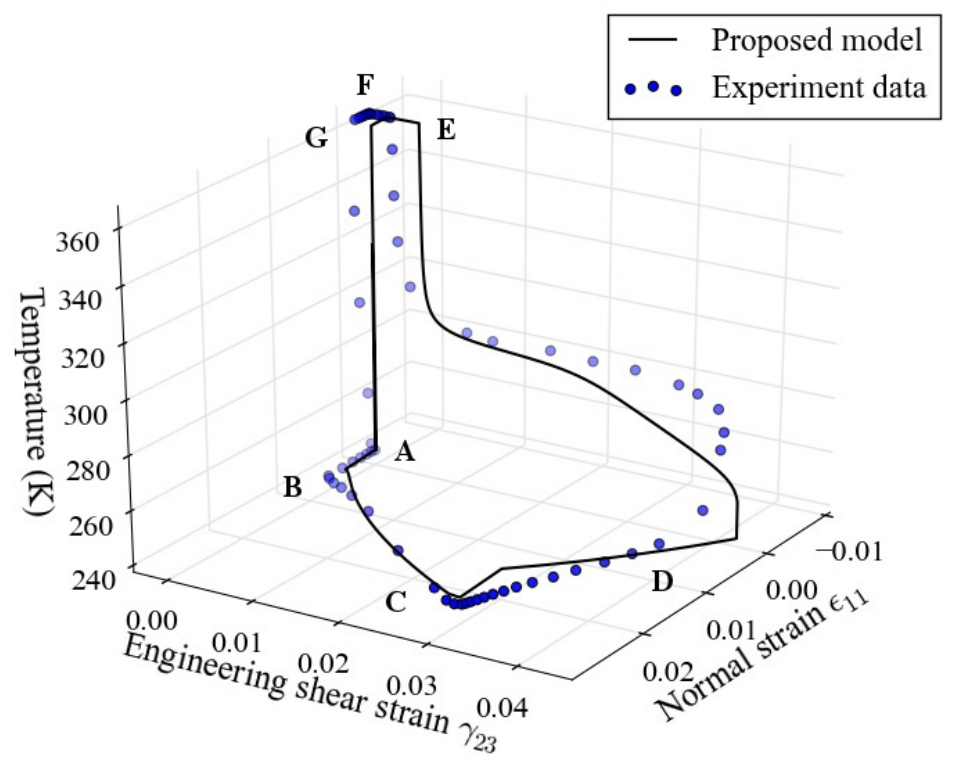

Figure 6: Simulated behavior (line) of the complex thermomechanical loading of NiTi tube and comparison with experimental data (points).

allowing for more elastic strain to appear at higher values of MVF. The austenitic modulus is recovered during reverse transformation. Note that such parameter identification

415 comes directly ou of an optimization process aimed at minimising the square difference between experimental and numerical strains. Also, the proposed model can be viewed here as an extension of Lagoudas et al (2012) model in the sense that it is a special case of the proposed model in absence of reorientation mechanism or combined forward/reverse transformation.

420 5.3. Tension-compression-torsion loading with ranging temperature

A more complicated loading path is chosen to put the new model under test. In (Grabe and Bruhns, 2009), an experiment alternating the stress level of the SMA material between tension and compression, combined with torsion, subjected to a wide range of temperature levels is presented. The full path is presented in Fig. 5. A tube specimen made of 50.7 at. \% NiTi with a wall thickness of $1.68 \mathrm{~mm}$ is heated from 193 to $243 \mathrm{~K}$ at a stress-free state. It is then loaded in the axial direction to $120 \mathrm{MPa}$ in tension up to point $\mathrm{B}$, and then to a combined tension-torsion state at $120 \mathrm{MPa}$ in torsion to point $\mathrm{C}$. From this point, axial stress is reversed to $132 \mathrm{MPa}$ in compression (D). Then, maintaining the stresses, the tube is heated to $363 \mathrm{~K}(\mathrm{E})$, where it is gradially released from torsion first

${ }_{430}(\mathrm{~F})$ and then compression $(\mathrm{G})$. Finally, the specimen is cooled down to $243 \mathrm{~K}$, returning to the initial point $\mathrm{A}$. The identified material parameters appear in Table 6 . This time, the model parameters for asymmetry are important, since compression is present in the loading path.

The curves resulting from the loading simulation are presented in Figs. 6 and 7 and compared with the reported experiment results. The material behavior in the space of 


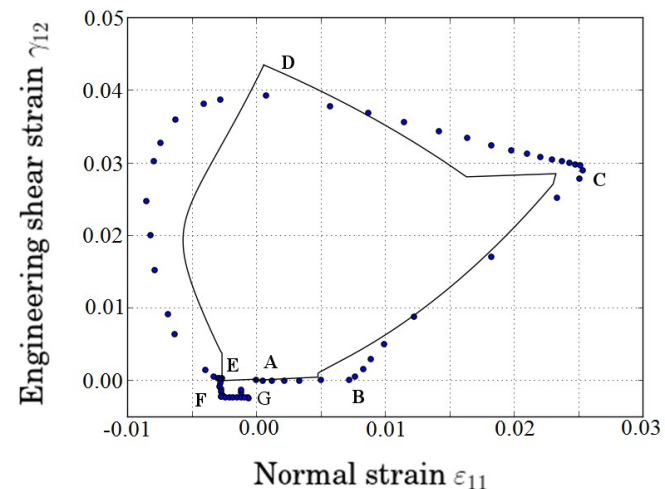

(a) Shear - axial strain relation

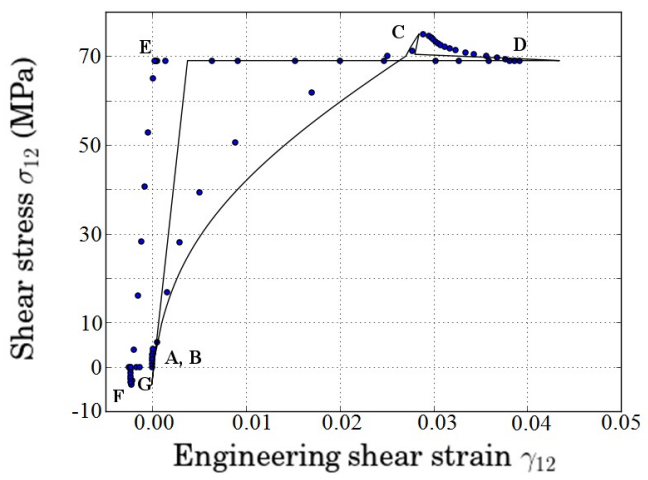

(c) Shear stress - strain relation

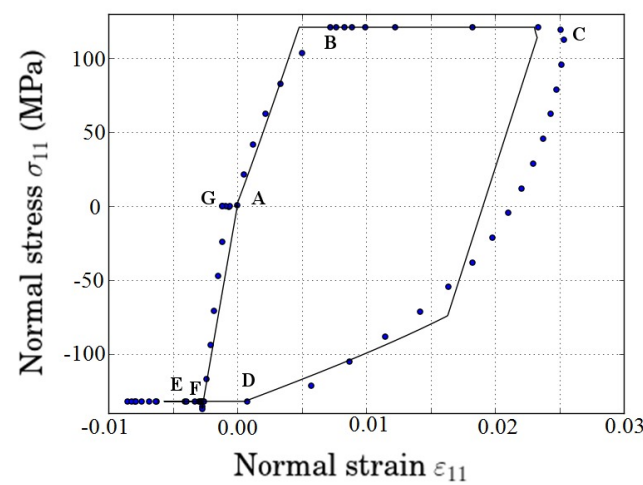

(b) Normal stress - strain relation

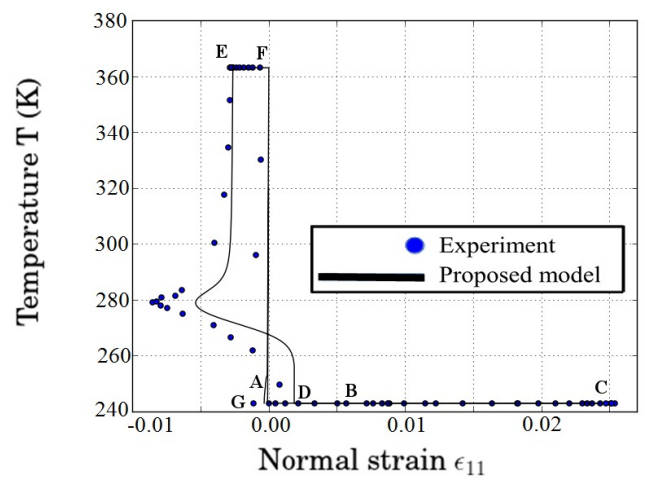

(d) Temperature - axial strain relation

Figure 7: Experimental results (points) and model reponse (line) on SMA thin-walled tube from Grabe and Bruhns 2009

temperature, axial and shear strain is presented in the 3D graph in Fig. 6 . In Figs. 7 (a) to (d), the relation between the material variables is outlined during the history of the experiment. In the loading history up to point $\mathrm{D}$, all non-linear behavior is due to the orientation mechanism. The temperature is below the martensite transition points and therefore no transformation is allowed. The two-branch response in the C-D path in the normal stress-strain curve confirms the kinematic character of the hardening during reorientation. However, the discrepancies between experiment and simulation imply that a non-linear hardening law would be better suited.

In the part D-E of the loading path, where the tube is heated and submitted to reverse transformation, the current model handles the change in the direction of transformation strains, as revealed in the shear-axial strain curve. The idea of a simple recovery of transformation strains would lead to straight line from point D to E in Fig. 7 (a) and (d). The form of the selected evolution law of (25) however, in combination with the simultaneous activation of reorientation, imposes a more complex strain path resembling the experimental data. 


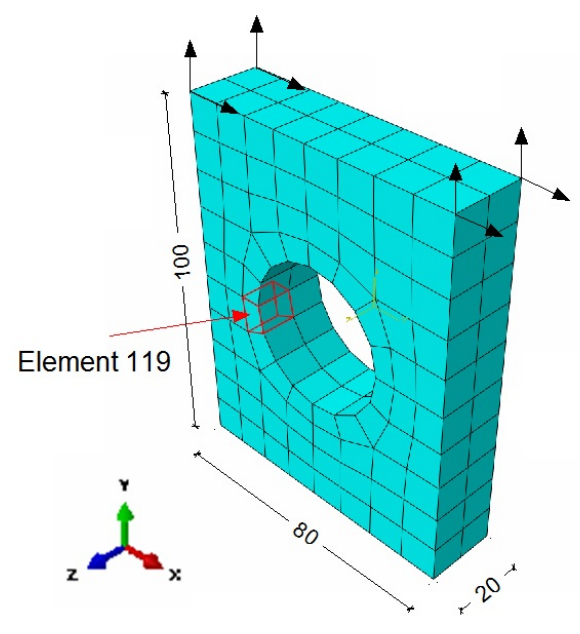

(a) Geometry of the pierced plate

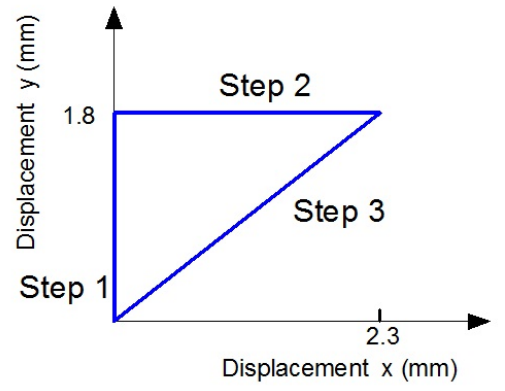

(b) Loading path for the top surface

Figure 8: Geometry (a) and loading history (b) of the holed plate. Dimensions in mm.

\subsection{Analysis of strain rate dependency on SMA structure}

The effect of themomechanical coupling becomes evident in cases when the strain rate imposed by external working is so high that regular convection is not sufficient

455 to ensure isothermal conditions. In a simple test-case scenario, a holed plate made of NiTi is simulated using the Finite Element Package (FEA) Abaqus, and subjected to non-proportional mechanical loading. Convection-type thermal boundary conditions are applied to the surface of the structure.

The plate is considered to be placed in a temperature pool, in which the air has a con-

${ }_{460}$ stant temperature $T_{\infty}=280 \mathrm{~K}$. A "surface film interaction" with convection parameter $\bar{h}=0.4 \mathrm{~kW} / \mathrm{m}^{2} K($ Enemark et al, 2015, Pathak et al 2010) is kept constant throughout the whole analysis to control heat exchanges. Such boundary conditions ressemble to that of a real experiment at room temperature. The material properties are reported in Table 7. The bottom surface of the plate is considered in encastre conditions. In the first

465 step of the loading, tension is applied by controlling the position of the upper surface, gradually increasing to $1.8 \mathrm{~mm}$. Then, in the second step, a "shear-type" loading is applied, consisting of a uniform displacement applied on the same surface in the lateral direction, gradually reaching $2.3 \mathrm{~mm}$, while longitudinal displacement is maintained at the previous level. In the third step, the two displacements are simultaneously removed until full unloading is reached. The geometry and the history of loading is illustrated in Fig. 8. The full geometry has been simulated, since the thermomechanical load does not present any symmetry. C3D8T elements were used for the mesh. Two simulations were executed, where the boundary conditions of the top surface were imposed under two different speeds $\frac{\Delta l / l}{\Delta t}$ : at $10^{-5} \mathrm{sec}^{-1}$, considered to correspond to a "static" load,

475 and at $10^{-4} \mathrm{sec}^{-1}$, which induces a temperature change actively affecting the mechanical behavior. 


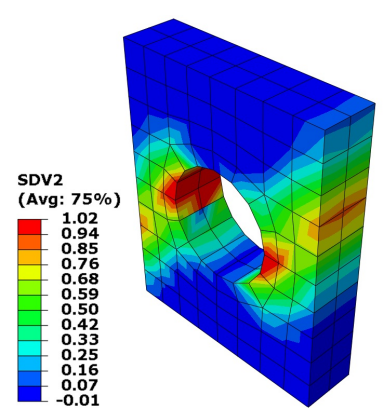

(a) Static speed, $\xi$ at Step 2

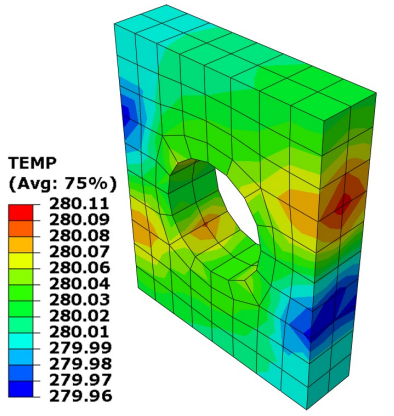

(d) Static, temp. at Step 2

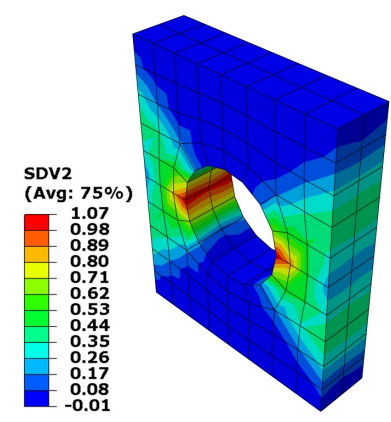

(b) Speed $10 x, \xi$ at Step 1

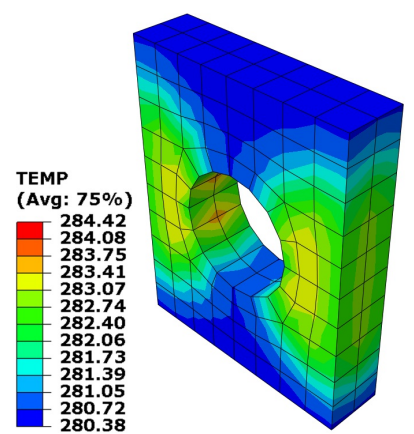

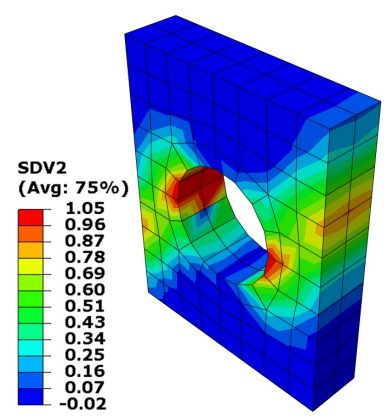

(c) Speed 10x, $\xi$ at Step 2

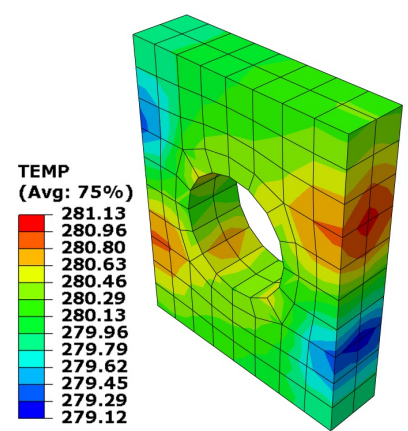

(e) Speed 10x, temp. at Step 1 (f) Speed 10x, temp. at Step 2

Figure 9: Martensitic volume fraction $(\xi)$ on the first row (a-c) and temperature contour maps (d-f) corresponding to the same time step for the two loading speeds at the end of Step 1 and end of Step 2. 1

On Fig. 9 it is clear that the thermomechanical coupling induces a significant change in the temperature field in the case of the high loading rate. The mechanical response of the material should therefore be impacted. Also, reorientation appears in the stress

480 concentration zone around the hole during loading step 2. It is particularly enlightening to look closer at this location and in the centroid of element 119 precisely. Undergoing reorientation, this material point has a particularly complex evolution of stress state, presented in Fig. 10. The equivalent Mises stress - Mises strain diagram provides us a clear view that the heat generation is actually dominated by phase transformation.

485 The crystallographic transition between austenite and martensite induces latent heat and dissipation. Such phenomena constitute a more important internal heat source compared to reorientation, the latter releasing heat through dissipation only.

The temperature maps on Figs. 9e and (9f) corresponding to the end of Steps 1 and 2 respectively exhibit a cooling effect deriving from the interaction with the ambient air. The reorientation present in Step 2 does not produce as much energy as forward transformation and therefore the air has the time to absorb a fraction of the excessive heat. In the corresponding volume fraction maps $(9 b)$ and $(9 c)$, the difference between the transformed zones is small enough to justify the lack of supplementary heat during Step 2. Still, the difference in temperature at the end of Step 2 between the static 


\begin{tabular}{lllllll}
\hline$E^{A}(\mathrm{MPa})$ & $E^{M}(\mathrm{MPa})$ & $v^{A}$ & $v^{M}$ & $\alpha\left(K^{-1}\right)$ & $H_{\text {min }}$ & $H_{\text {sat }}$ \\
50120 & 27000 & 0.30 & 0.30 & $10^{-5}$ & 0.0 & 0.056 \\
$k$ & $C^{A}(\mathrm{MPa} / \mathrm{K})$ & $C^{M}(\mathrm{MPa} / \mathrm{K})$ & $M_{f}(\mathrm{~K})$ & $M_{s}(\mathrm{~K})$ & $A_{s}(\mathrm{~K})$ & $A_{f}(\mathrm{~K})$ \\
0.021 & 6 & 6 & 253 & 258 & 272 & 277 \\
$Y_{o}^{r e o}(\mathrm{MPa})$ & $\varepsilon_{\max }^{r e}$ & $H^{r e}$ & $\mathrm{~b}$ & $\mathrm{n}$ & $\rho\left(k g / m^{3}\right)$ & $k_{t h}(W / m K)$ \\
120 & 0.05 & 1000 & 0 & $(2)$ & 6450 & 12 \\
\hline
\end{tabular}

Table 7: Material constants for NiTi plate subjected in non-proportional loading modeled in finite element analysis. $\rho$ is the mass density and $k_{t h}$ is the thermal conductivity constant.

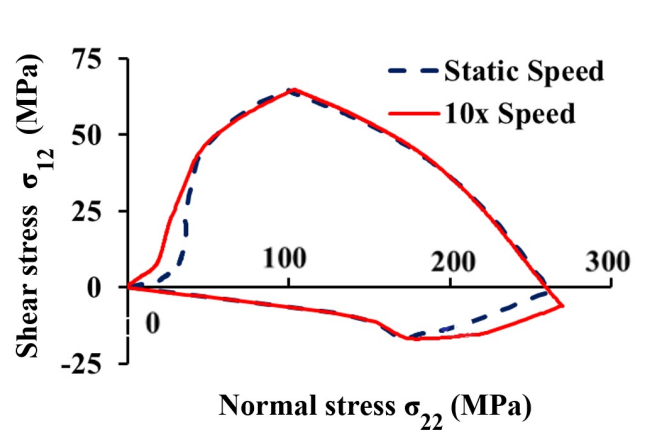

(a) History of $\sigma_{22}$ and $\sigma_{12}$ stresses

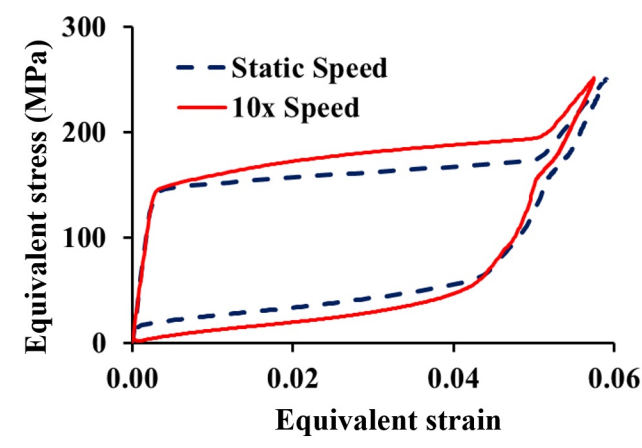

(b) Equivalent stress-strain diagram

Figure 10: Evolution of characteristic stress components (a) and of the equivalent (Von Mises) stress-strain relation (b) for the two loading rates for the element 119 of the mesh.

495 loading case $9 \mathrm{~d}$ and the loading at higher speed is evident, although the MVF contours are practically the same.

\section{Conclusion}

A new phenomenological model to describe the complex behavior of SMAs while experiencing coupled transformation/reorientation during complex, non-proportional thermomechanical loading is proposed. It has been shown that this model is thermodynamically consistent, and the proposed evolution equations correctly describe the evolution of martensitic volumes in a SMA. The original contribution of this work concerns the independent way in which the physical mechanisms are conceived to take place. The first contribution brought about is the decoupling of forward and reverse transformation. In such a way, it is observed that, in terms of modeling, these two can occur simultaneously inside the material. One more novelty is the simple form for describing the process of reorientation. It is also considered an independent mechanism. Finally, a general framework to resolve the numerical problem of complex non-proportional loading is presented

\footnotetext{
${ }^{1}$ Values of $\xi$ over 1 and below 0 are due to extrapolation from integration points to the element surface.
} 
for the case of multiple mechanisms. The thermomechanical effects are fully taken in

510 mind. Simulations of simple and complex experiments on SMA structures appearing in the literature have been carried out. The effect of strain rate in a pertinent finite element simulation has also been examined. In particular, the coupling between transformation and orientation allows to describe with a good accuracy the thermomechanical response of SMAs observed by several authors. The comparison of simulation with experimental

515 results is a finding that demonstrates the advanced predictive capability of the model to capture the complex overall thermomechanical behavior of SMAs.

\section{Acknowledgements}

The authors would like to extend their sincere gratitude to Dr. Otto T. Bruhns for providing with the full measurements corresponding to the experiment presented in 520 (Grabe and Bruhns, 2009) and utilized in the current work.

\section{References}

Ameduri S, Antonucci V, Artioli E, Asprone D, Auricchio F, Barbarino S, Boatti E, Conti M, Dimino I, Faiella G, Marfia S, Martone A, Menna C, Pecora R, Sacco E, Stortiero F, Vigliotti A, Villa E (2015) Shape Memory Alloy Engineering. Elsevier, DOI 10.1016/B978-0-08-099920-3.01002-0, URL 525 http://www.sciencedirect.com/science/article/pii/B9780080999203010020

Arghavani J, Auricchio F, Naghdabadi R, Reali A, Sohrabpour S (2010) A 3-D phenomenological constitutive model for shape memory alloys under multiaxial loadings. International Journal of Plastic-

n ity 26(7):976-991, DOI 10.1016/j.ijplas.2009.12.003, URL http://www.sciencedirect.com/science/ article/pii/S0749641909001648

530 Auricchio F, Bonetti E, Scalet G, Ubertini F (2014) Theoretical and numerical modeling of shape memory alloys accounting for multiple phase transformations and martensite reorientation. International

Journal of Plasticity 59:30-54, DOI 10.1016/j.ijplas.2014.03.008, URL http://www.sciencedirect. com/science/article/pii/S0749641914000515

Auricchio F, Boatti E, Conti M (2015) Chapter 12 - SMA Cardiovascular Applications and Computer-Based Design. In: Shape Memory Alloy Engineering, Elsevier, pp 343-367, DOI

17 10.1016/B978-0-08-099920-3.00012-7, URL http://www.sciencedirect.com/science/article/pii/ B9780080999203000127

Barbarino S, Saavedra Flores EI, Ajaj RM, Dayyani I, Friswell MI (2014) A review on shape memory alloys with applications to morphing aircraft. Smart Materials and Structures 23(6):063,001, DOI

540 10.1088/0964-1726/23/6/063001, URL http://iopscience.iop.org/article/10.1088/0964-1726/ $23 / 6 / 063001$

Bouvet C, Calloch S, Lexcellent C (2004) A phenomenological model for pseudoelasticity of shape memory alloys under multiaxial proportional and nonproportional loadings. European Journal of Mechanics - A/Solids 23(1):37-61, DOI 10.1016/j.euromechsol.2003.09.005, URL http://www.sciencedirect. com/science/article/pii/S0997753803001104

Boyd JG, Lagoudas DC (1994) Thermomechanical Response of Shape Memory Composites. Journal of Intelligent Material Systems and Structures 5(3):333-346, DOI 10.1177/1045389X9400500306, URL http://jim. sagepub.com/content/5/3/333. short

Brinson L (1993) One-Dimensional Constitutive Behavior of Shape Memory Alloys: Thermomechanical Derivation with Non-Constant Material Functions and Redefined Martensite Internal Variable. Journal of Intelligent Material Systems and Structures 4(2):229-242, DOI 10.1177/1045389X9300400213, URL http://jim.sagepub.com/content/4/2/229.abstract

Brinson L, Schmidt I, Lammering R (2004) Stress-induced transformation behavior of a polycrystalline NiTi shape memory alloy: micro and macromechanical investigations via in situ optical microscopy

555 Journal of the Mechanics and Physics of Solids 52(7):1549-1571, DOI 10.1016/j.jmps.2004.01.001, URL http://www.sciencedirect.com/science/article/pii/S0022509604000079 
Chatziathanasiou D, Chemisky Y, Meraghni F, Chatzigeorgiou G, Patoor E (2015) Phase Transformation of Anisotropic Shape Memory Alloys: Theory and Validation in Superelasticity. Shape Memory and Superelasticity 1(3):359-374, DOI 10.1007/s40830-015-0027-y, URL http://link.springer.com/ article/10.1007/s40830-015-0027-y/fulltext.html\{\#\}CR25

Chatzigeorgiou GE, Lagoudas DC (2009) < title $>$ A constitutive model for high temperature SMAs exhibiting viscoplastic behavior $</$ title $>$. In: Ounaies Z, Li J (eds) SPIE Smart Structures and Materials + Nondestructive Evaluation and Health Monitoring, International Society for Optics and Photonics, 1. pp 72,890U-72,890U-7, DOI 10.1117/12.817689, URL http://proceedings.spiedigitallibrary. org/proceeding . aspx?articleid=1337287

Chemisky Y, Duval A, Patoor E, Ben Zineb T (2011) Constitutive model for shape memory alloys including phase transformation, martensitic reorientation and twins accommodation. Mechanics of

Materials 43(7):361-376, DOI 10.1016/j.mechmat.2011.04.003, URL http://dx.doi.org/10.1016/j. mechmat.2011.04.003

570 Chemisky Y, Chatzigeorgiou G, Kumar P, Lagoudas DC (2014) A constitutive model for cyclic actuation of high-temperature shape memory alloys. Mechanics of Materials 68:120-136,

DOI 10.1016/j.mechmat.2013.07.020, URL http://www.sciencedirect.com/science/article/pii/ S0167663613001518

Chemisky Y, Meraghni F, Bourgeois N, Cornell S, Echchorfi R, Patoor E (2015) Analysis of the de-

575 formation paths and thermomechanical parameter identification of a shape memory alloy using digital image correlation over heterogeneous tests. International Journal of Mechanical Sciences 96-97:13-24, DOI 10.1016/j.ijmecsci.2015.03.007, URL http://www.sciencedirect.com/science/ article/pii/S0020740315000831

Cisse C, Zaki W, Zineb T (2015) A review of constitutive models and modeling techniques for shape memory alloys. International Journal of Plasticity 76:244-284, DOI 10.1016/j.ijplas.2015.08.006, URL http://www.sciencedirect.com/science/article/pii/S0749641915001436

Coleman B, Gurtin M (1967) Thermodynamics with internal variables. J Chem Phys 47(2):85-98

Coleman B, Noll W (1963) The thermodynamics of elastic materials with heat conduction and viscocity. Arch Rational Mech Anal 13:167

585 Enemark S, Savi MA, Santos IF (2015) Experimental analyses of dynamical systems involving shape memory alloys. Smart Structures and Systems 15(6):1521-1542, DOI 10.12989/sss.2015.

15.6.1521, URL http://121.183.206.200:8080/proto.board/abstractArticleContentView?page= article $\{\&\}$ journal=sss $\{\&\}$ volume $=15\{\&\}$ num $=6\{\&\}$ ordernum $=7\{\&\}$ s ite=korsc

Grabe C, Bruhns O (2009) Path dependence and multiaxial behavior of a polycrystalline NiTi alloy within the pseudoelastic and pseudoplastic temperature regimes. International Journal of Plastic-

n ity 25(3):513-545, DOI 10.1016/j.ijplas.2008.03.002, URL http://www.sciencedirect.com/science/ article/pii/S0749641908000387

Grolleau V, Louche H, Delobelle V, Penin A, Rio G, Liu Y, Favier D (2011) Assessment of tension-compression asymmetry of NiTi using circular bulge testing of thin plates. Scripta Mate-

595 rialia 65(4):347-350, DOI 10.1016/j.scriptamat.2011.05.003, URL http://www.sciencedirect.com/ science/article/pii/S1359646211002648

Hartl DJ, Lagoudas D (2009) Constitutive modeling and structural analysis considering simultaneous phase transformation and plastic yield in shape memory alloys. Smart Materials and Structures

[1 18(10):104,017, DOI 10.1088/0964-1726/18/10/104017, URL http://iopscience.iop.org/article/ $600 \quad 10.1088 / 0964-1726 / 18 / 10 / 104017$

Hartl DJ, Lagoudas DC (2007) Aerospace applications of shape memory alloys. Proceedings of the Institution of Mechanical Engineers, Part G: Journal of Aerospace Engineering 221(4):535-552, DOI 10.1243/09544100JAERO211, URL http://pig.sagepub.com/content/221/4/535.abstract

Hartl DJ, Lagoudas DC (2008) Thermomechanical Characterization of Shape Memory Alloy Materials. In: Shape Memory Alloys, Springer US, Boston, MA, pp 53-119, DOI 10.1007/978-0-387-47685-8, URL http: //www . springerlink. com/index/10.1007/978-0-387-47685-8

Hartl DJ, Chatzigeorgiou G, Lagoudas DC (2010) Three-dimensional modeling and numerical analysis of rate-dependent irrecoverable deformation in shape memory alloys. International Journal of Plasticity

26(10):1485-1507, DOI 10.1016/j.ijplas.2010.01.002, URL http://www.sciencedirect.com/science/ article/pii/S0749641910000033

Helm D, Haupt P (2003) Shape memory behaviour: modelling within continuum thermomechanics International Journal of Solids and Structures 40(4):827-849, DOI 10.1016/S0020-7683(02)00621-2, URL http://www . sciencedirect.com/science/article/pii/S0020768302006212

Juhász L, Andrä H, Hesebeck O (2001) A Simple Model for Shape Memory Alloys Under Multi-axial 615 Non-Proportional Loading. In: Smart Materials, pp 51-65 
Khandelwal A, Buravalla V (2009) Models for Shape Memory Alloy Behavior: An overview of modeling approaches. URL https://journals.tdl.org/ijscs/index.php/ijscs/article/view/2318

Lagoudas D (2008) Shape Memory Alloys - Modeling and Engineering Applications. Springer, URL http://www. springer.com/materials/book/978-0-387-47684-1

620 Lagoudas D, Hartl D, Chemisky Y, Machado L, Popov P (2012) Constitutive model for the numerical analysis of phase transformation in polycrystalline shape memory alloys. International Journal of Plasticity 32-33(null):155-183, DOI 10.1016/j.ijplas.2011.10.009

Lagoudas DC, Entchev PB, Popov P, Patoor E, Brinson LC, Gao X (2006) Shape memory alloys, Part II: Modeling of polycrystals. Mechanics of Materials 38(5-6):430-462, DOI 10.1016/j.mechmat.2005. 08.003, URL http://www.sciencedirect.com/science/article/pii/S0167663605001201

Lecce L, Concilio A (eds) (2014) Shape Memory Alloy Engineering: For Aerospace, Structural and Biomedical Applications. URL https://books.google.com/books?hl=el $\{\&\} l r=\{\&\} i d=$ bwBOAwAAQBA J $\{\&\}$ pgis $=1$

Lemaitre J, Chaboche JL (2002) Mechanics of Solid Materials. Cambridge University Press, 630 URL http://books.google.co.in/books/about/Mechanics\{_\}of\{_\}Solid\{_\}Materials.html? id=YfHTNHfjqXoC $\{\&\}$ pgis $=1$

Lexcellent C, Boubakar M, Bouvet C, Calloch S (2006) About modelling the shape memory alloy behaviour based on the phase transformation surface identification under proportional loading and anisothermal conditions. International Journal of Solids and Structures 43(3-4):613-

635 626, DOI 10.1016/j.ijsolstr.2005.07.004, URL http://www.sciencedirect.com/science/article/ pii/S0020768305004361

Liu Y, Favier D (2000) Stabilisation of martensite due to shear deformation via variant reorientation

in polycrystalline NiTi. Acta Materialia 48(13):3489-3499, URL http://www.sciencedirect.com/ science/article/pii/S1359645400001294

640 Lubliner J (1972) On the thermodynamic foundations of non-linear solid mechanics. International Journal of Non-linear Mechanics 7:237-254

Luig P, Bruhns O (2008) On the modeling of shape memory alloys using tensorial internal variables

Materials Science and Engineering: A 481-482:379-383, DOI 10.1016/j.msea.2007.03.123, URL http: //www.sciencedirect.com/science/article/pii/S0921509307012865

645 Meraghni F, Chemisky Y, Piotrowski B, Echchorfi R, Bourgeois N, Patoor E (2014) Parameter identification of a thermodynamic model for superelastic shape memory alloys using analytical calculation of the sensitivity matrix. European Journal of Mechanics - A/Solids 45:226-237, DOI 10.1016/j.euromechsol. 2013.12.010, URL http://www.sciencedirect.com/science/article/pii/S0997753813001708

Merzouki T, Collard C, Bourgeois N, Ben Zineb T, Meraghni F (2010) Coupling between measured kinematic fields and multicrystal SMA finite element calculations. Mechanics of Materials 42(1):72-95,

1. DOI 10.1016/j.mechmat.2009.09.003, URL http://www.sciencedirect.com/science/article/pii/ S0167663609001562

Mohd Jani J, Leary M, Subic A, Gibson MA (2014) A review of shape memory alloy research, applications and opportunities. Materials \& Design 56:1078-1113, DOI 10.1016/j.matdes.2013.11.084, URL http: //www.sciencedirect.com/science/article/pii/S0261306913011345

Morgan N (2004) Medical shape memory alloy applications-the market and its products. Materials Science and Engineering: A 378(1-2):16-23, DOI 10.1016/j.msea.2003.10.326, URL http: //www.sciencedirect.com/science/article/pii/S0921509303015132

Morin C, Moumni Z, Zaki W (2011) Thermomechanical coupling in shape memory alloys under cyclic loadings: Experimental analysis and constitutive modeling. International Journal of Plasticity

27(12):1959-1980, DOI 10.1016/j.ijplas.2011.05.005, URL http://www.sciencedirect.com/science/ article/pii/S0749641911000817

Otsuka K, Wayman CM (1999) Shape Memory Materials. Cambridge University Press, URL https: //books.google.com/books?hl=en $\{\&\} l r=\{\&\}$ id=DvItE9XUlN8C $\{\&\}$ pg is $=1$

665 Panico M, Brinson L (2007) A three-dimensional phenomenological model for martensite reorientation in shape memory alloys. Journal of the Mechanics and Physics of Solids 55(11):2491-2511, DOI 10.1016 j.jmps.2007.03.010, URL http://www.sciencedirect.com/science/article/pii/S0022509607000713

Pathak A, Brei D, Luntz J (2010) Transformation strain based method for characterization of convective heat transfer from shape memory alloy wires. Smart Materials and Structures 19(3):035,005,

670 DOI 10.1088/0964-1726/19/3/035005, URL http://inis.iaea.org/Search/search.aspx?orig\{_\}q= RN $: 44126018$

Patoor E, Berveiller M (1997) Micromechanical modelling of the thermomechanical behaviour of shape memory alloys. In: Fishcer F, Berveiller M (eds) Mechanics of Solids with Phase Change, Springer Verlag, Wien, pp 121-188 
675 Patoor E, El Amrani M, Eberhardt A, Berveiller M (1995) Determination of the origin for the dissymmetry observed between tensile and compression tests on shape memory alloys. J Phys IV(2):495-500

Patoor E, Lagoudas DC, Entchev PB, Brinson LC, Gao X (2006) Shape memory alloys, Part I: General properties and modeling of single crystals. Mechanics of Materials 38(5-6):391-429, DOI 10.1016/j.mechmat.2005.05.027, URL http://ww.sciencedirect.com/science/article/pii/ S0167663605001195

Peultier B, Ben Zineb T, Patoor E (2008) A simplified micromechanical constitutive law adapted to the design of shape memory applications by finite element methods. Materials Science and Engineering: A 481-482(null):384-388, DOI 10.1016/j.msea.2007.05.117

Peyroux R, Chrysochoos A, Licht C, Löbel M (1998) Thermomechanical couplings and pseudoe685 lasticity of shape memory alloys. International Journal of Engineering Science 36(4):489-509,

DOI 10.1016/S0020-7225(97)00052-9, URL http://www.sciencedirect.com/science/article/pii/ S0020722597000529

Popov P, Lagoudas DC (2007) A 3-D constitutive model for shape memory alloys incorporating pseudoelasticity and detwinning of self-accommodated martensite. International Journal of Plas-

690 ticity 23(10-11):1679-1720, DOI 10.1016/j.ijplas.2007.03.011, URL http://www.sciencedirect.com/ science/article/pii/S0749641907000460

Qidwai M, Lagoudas D (2000) On thermomechanics and transformation surfaces of polycrystalline NiT shape memory alloy material. International Journal of Plasticity 16(10-11):1309-1343, DOI 10.1016 / S0749-6419(00)00012-7, URL http://dx.doi.org/10.1016/S0749-6419(00)00012-7

695 Saint-Sulpice L, Chirani SA, Calloch S (2009) A 3D super-elastic model for shape memory alloys taking into account progressive strain under cyclic loadings. Mechanics of Materials 41(1):12-

1 26, DOI 10.1016/j.mechmat.2008.07.004, URL http://linkinghub.elsevier.com/retrieve/pii/ S0167663608001051

Saleeb A, Arnold S, Castelli M, Wilt T, Graf W (2001) A general hereditary multimechanism-based deformation model with application to the viscoelastoplastic response of titanium alloys. Inter1. national Journal of Plasticity 17(10):1305-1350, DOI 10.1016/S0749-6419(00)00086-3, URL http: //www.sciencedirect.com/science/article/pii/S0749641900000863

Saleeb A, Padula S, Kumar A (2011) A multi-axial, multimechanism based constitutive model for the comprehensive representation of the evolutionary response of SMAs under general thermomechanica loading conditions. International Journal of Plasticity 27(5):655-687, DOI 10.1016/j.ijplas.2010.08. 012, URL http://www.sciencedirect.com/science/article/pii/S0749641910001178

Sedlák P, Frost M, Benešová B, Ben Zineb T, Sittner P (2012) Thermomechanical model for NiTibased shape memory alloys including R-phase and material anisotropy under multi-axial loadings.

International Journal of Plasticity 39(null):132-151, DOI 10.1016/j.ijplas.2012.06.008, URL http:

710 //www.sciencedirect.com/science/article/pii/S0749641912001027

Sittner P, Hara Y, Tokuda M (1995) Experimental study on the thermoelastic martensitic transformation in shape memory alloy polycrystal induced by combined external forces. Metallurgical and Materials Transactions A 26(11):2923-2935, DOI 10.1007/BF02669649, URL http://link.springer.com/10. $1007 /$ BF02669649

715 Sittner P, Heller L, Pilch J, Sedlak P, Frost M, Chemisky Y, Duval A, Piotrowski B, Ben Zineb T, Patoor E, Auricchio F, Morganti S, Reali A, Rio G, Favier D, Liu Y, Gibeau E, Lexcellent C, Boubakar L, Hartl D, Oehler S, Lagoudas D, Humbeeck JV (2009) Roundrobin SMA modeling. In: ESOMAT 2009 - 8th European Symposium on Martensitic Transformations, EDP Sciences, Les Ulis, France, p 08001, DOI 10.1051/esomat/200908001, URL http://dx.doi.org/10.1051/esomat/200908001

720 Souza A, Mamiya E, Zouain N (1998) Three-dimensional model for solids undergoing stress-induced phase transitions. European Journal of Mechanics - A/Solids1 17:789-806

Taillard K, Chirani SA, Calloch S, Lexcellent C (2008) Equivalent transformation strain and its relation with martensite volume fraction for isotropic and anisotropic shape memory alloys. Mechanics of Materials 40(4-5):151-170, DOI 10.1016/j.mechmat.2007.07.005

725 Van Humbeeck J (1999) Non-medical applications of shape memory alloys. Materials Science and Engineering: A 273-275:134-148, DOI 10.1016/S0921-5093(99)00293-2, URL http://www.sciencedirect. com/science/article/pii/S0921509399002932 


\section{Appendix A. Mathematical formulation of the evolution equation for trans- formation strain}

730 Revisiting equation 11, a rigorous mathematical formalism is intended to be given. The moving boundaries of the martensite volume, the mathematical representation of which is found in the term $\dot{V}_{M}$ is shown to be an important aspect to differentiate the two mechanisms. Therefore, it is essential to define the rate of change of the martensitic volume.

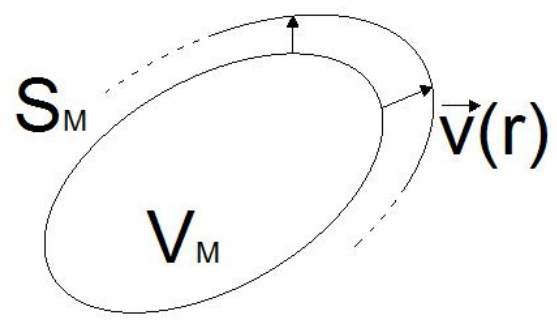

Figure A.11: Moving boundary of martensitic volume

In Fig. A.11 $S_{M}$ is the boundary surface between the martensite and the austenite volumes. The points on this surface have coordinates $\vec{X}(r)$.

$\vec{v}(r)=\dot{\vec{X}}$ is the velocity with which the boundary moves. Here, a flux of martensitic volume is envisioned, basically martensitic volume leaking out of the martensitic volume that is already there. The total difference of it in a step of time should be:

$$
d V_{M}=\int_{V_{M}} \operatorname{div}(d \vec{X}(r)) d V
$$

and in rate form:

$$
\dot{V}_{M}=\int_{V_{M}} \operatorname{div} \dot{\vec{X}}(r) d V=\int_{V_{M}} \operatorname{div} \vec{v}(r) d V
$$

${ }_{735}$ On the other hand, the rate of the mean transformation strain is given strictly by:

$$
\begin{aligned}
\dot{\bar{\varepsilon}}^{\boldsymbol{T}}= & \left(\frac{1}{V_{M}} \int_{V_{M}} \tilde{\varepsilon}_{r}^{\boldsymbol{T}} d V\right)=-\frac{\dot{V}_{M}}{V_{M}^{2}} \int_{V_{M}} \tilde{\varepsilon}_{r}^{\boldsymbol{T}} d V+\frac{1}{V_{M}}\left(\int_{V_{M}} \tilde{\varepsilon}_{r}^{\boldsymbol{T}} d V\right)= \\
& =-\frac{\dot{V}_{M}}{V_{M}} \bar{\varepsilon}^{\boldsymbol{T}}+\frac{1}{V_{M}}\left(\int_{V_{M}} \tilde{\varepsilon}_{r}^{\boldsymbol{T}} d V\right)
\end{aligned}
$$

According to Leibniz-Reynolds' transport theorem, the rate of the last integral is 
written:

$$
\left(\int_{V_{M}} \tilde{\varepsilon}_{r}^{T} d V\right)=\int_{V_{M}}\left(\dot{\tilde{\varepsilon}}_{r}^{T}+\tilde{\varepsilon}_{r}^{T} \operatorname{div} \vec{v}(r)\right) d V=\int_{V_{M}} \dot{\tilde{\varepsilon}}_{r}^{T} d V+\int_{V_{M}}\left(\tilde{\varepsilon}_{r}^{T} \operatorname{div} \vec{v}(r)\right) d V
$$

$\vec{v}(r)$ being the vector defined earlier.

In the light of equation 12 :

$$
\left(\int_{V_{M}} \tilde{\varepsilon}_{r}^{\boldsymbol{T}} d V\right)=V_{M} \dot{\bar{\varepsilon}}^{r e}+\int_{V_{M}}\left(\tilde{\varepsilon}_{r}^{\boldsymbol{T}} \operatorname{div} \vec{v}(r)\right) d V
$$

Here, according to the second mean value theorem for integrals, there is a point $\psi$ on the surface $S_{M}$ for which:

$$
\int_{V_{M}}\left(\tilde{\varepsilon}_{r}^{T} \operatorname{div} \vec{v}(r)\right) d V=\tilde{\varepsilon}_{\psi}^{T} \int_{V_{M}} \operatorname{div} \vec{v}(r) d V
$$

and, by effect of A.1):

$$
\int_{V_{M}}\left(\tilde{\varepsilon}_{r}^{T} \operatorname{div} \vec{v}(r)\right) d V=\varepsilon^{T}(\psi) \dot{V}_{M}
$$

The value of $\boldsymbol{\varepsilon}^{\boldsymbol{T}}(\psi)$ is the weighted average of $\tilde{\varepsilon}_{\boldsymbol{r}}^{\boldsymbol{T}}$ based on $\operatorname{div} \vec{v}(r)$ in the volume $V_{M}$. Here, the definition of $\overline{\boldsymbol{\Lambda}}^{\boldsymbol{T}}$ is updated in order to fit $\boldsymbol{\varepsilon}^{\boldsymbol{T}}(\psi)$. It is the weighted average of ${ }_{740} \quad \tilde{\varepsilon}_{r}^{T}$ on the surface $S_{M}$ based on the divergence of the velocity in $V_{M}$ :

$$
\overline{\boldsymbol{\Lambda}}^{\boldsymbol{T}}=\frac{\int_{V_{M}}\left(\tilde{\varepsilon}_{r}^{\boldsymbol{T}} \operatorname{div} \vec{v}\right) d V}{\int_{V_{M}} \operatorname{div} \vec{v} d V}=\frac{\int_{V_{M}}\left(\tilde{\varepsilon}_{r}^{\boldsymbol{T}} \operatorname{div} \vec{v}\right) d V}{\dot{V}_{M}}
$$

Still, in this sense, the product

$$
\bar{\Lambda}^{T} \dot{V}_{M}=\int_{V_{M}}\left(\tilde{\varepsilon}_{r}^{T} \operatorname{div} \vec{v}\right) d V
$$

will represent, according to (A.4) the contribution of the transformation strain inside the newly formed martensitic volume to the rate of change of $\bar{\varepsilon}^{T}$.

Back to the equation A.3, it will be written, according to A.4 and A.6):

$$
\dot{\bar{\varepsilon}}^{\boldsymbol{T}}=-\frac{\dot{V}_{M}}{V_{M}} \bar{\varepsilon}^{\boldsymbol{T}}+\frac{1}{V_{M}}\left(V_{M} \dot{\bar{\varepsilon}}^{r e}+\overline{\boldsymbol{\Lambda}}^{\boldsymbol{T}} \dot{V}_{M}\right)=\left(\overline{\boldsymbol{\Lambda}}^{\boldsymbol{T}}-\bar{\varepsilon}^{\boldsymbol{T}}\right) \frac{\dot{V}_{M}}{V_{M}}+\dot{\bar{\varepsilon}}^{r e}
$$

and by effect of 10 .

$$
\dot{\bar{\varepsilon}}^{T}=\left(\bar{\Lambda}^{T}-\bar{\varepsilon}^{T}\right) \frac{\dot{\xi}}{\xi}+\dot{\bar{\varepsilon}}^{r e}
$$


which is the rate form of (11).

Substituting this in 3 it is found that:

$$
\dot{\varepsilon}^{T}=\dot{\xi} \bar{\Lambda}^{T}+\xi \dot{\bar{\varepsilon}}^{r e}
$$

745 In the incremental scheme described in Fig. 1. when an elemental part of martensite $d V_{M}$ is considered to be removed after the first step, it seems necessary to remove all the transformation strain existing within its volume. This is considered to have a mean value equal to the mean value existing in $V_{M}$. Therefore: $\bar{\Lambda}^{\boldsymbol{T}}=\bar{\varepsilon}^{\boldsymbol{T}}$. In the absence of reorientation: $\dot{\varepsilon}^{T}=\dot{\xi} \bar{\varepsilon}^{T}$

Still, there is the need for a major constraint of SMA behavior to be satisfied: inelastic strains return to zero when there is no martensitic volume. To this end, the following case of loading of a RVE is considered, which induces reverse transformation:

$\dot{\xi} \leq 0$ in a time frame $\Delta t=t-0$. The reverse transformation is concluded at $\mathrm{t}$, meaning that $\xi(t)=0$. $\dot{\bar{\varepsilon}}^{r e} \neq \mathbf{0}$ for at least one point of $\Delta t$, meaning that reorientation is not

755 excluded. Finally, it is assumed that $\bar{\Lambda}^{\boldsymbol{T}}=\bar{\varepsilon}^{\boldsymbol{T}}$, since there is no forward transformation.

It is deduced that:

$\dot{\bar{\Lambda}}^{T}=\dot{\bar{\varepsilon}}^{T}$ and, by effect of A.8):

$$
\dot{\bar{\varepsilon}}^{T}=\dot{\bar{\varepsilon}}^{r e}=\dot{\bar{\Lambda}}^{T}
$$

The equation $(3)$ is now:

$$
\dot{\varepsilon}^{T}=\dot{\xi} \bar{\Lambda}^{T}+\xi \dot{\bar{\Lambda}}^{T}=\left(\xi \dot{\bar{\Lambda}}^{T}\right)
$$

Looking for $\varepsilon^{\boldsymbol{T}}(t)$, it is found that:

$$
\varepsilon^{\boldsymbol{T}}(t)=\varepsilon^{\boldsymbol{T}}(0)+\int_{0}^{t} \dot{\varepsilon}^{\boldsymbol{T}}(\tau) d \tau=\varepsilon^{\boldsymbol{T}}(0)+\int_{0}^{t}\left(\xi \dot{\bar{\Lambda}}^{\boldsymbol{T}}\right) d \tau=\boldsymbol{\varepsilon}^{\boldsymbol{T}}(0)+\xi(t) \overline{\boldsymbol{\Lambda}}^{\boldsymbol{T}}(t)-\xi(0) \bar{\Lambda}^{\boldsymbol{T}}(0)
$$

and

$$
\varepsilon^{\boldsymbol{T}}(t)=\varepsilon^{\boldsymbol{T}}(0)+\xi(t) \bar{\varepsilon}^{\boldsymbol{T}}(t)-\xi(0) \bar{\varepsilon}^{\boldsymbol{T}}(0)=\varepsilon^{\boldsymbol{T}}(0)+0 \cdot \bar{\varepsilon}^{\boldsymbol{T}}(t)-\varepsilon^{\boldsymbol{T}}(0)=\mathbf{0}
$$

Conclusively, the finding that $\varepsilon^{\boldsymbol{T}}(t)=\mathbf{0}$ is easily reached. This is an indication that the assumptions made so far satisfy the physical limitations of reverse transformation.

\section{Appendix B: Implementation of thermomechanical effects with Abaqus}

Any computation associated with the heat caused by mechanical working is carried out during the loop of force balance. After any kind of computation, a value corresponding to a variable belongs to a set of three repetitive processes. The most general process is the one that loops all the steps required to complete a time step. It is called here "the n loop".

765 The second process is the one that loops the RVE to find the balance in forces. It is called "the $\omega$ loop".

The third process is the one that loops the algebraic manipulations to find the next admissible set of variable coordinates for a RVE. It is called "the k loop". 
When the $\mathrm{n}$ loop is carried out, a differential

$$
\Delta x^{n+1}=x^{n+1}-x^{n}
$$

is approximated to find $x^{n+1}$. Here, $x^{n(w+1)}$ is considered a constant. When the $\omega$ loop is carried out, a differential

$$
\delta x^{\omega+1}=x^{\omega+1}-x^{\omega}
$$

is approximated to find $x^{\omega+1}$. Here, $x^{n+1(\omega)}$ is considered a constant. When the $\mathrm{k}$ loop is carried out, a differential

$$
\tilde{\delta} x^{k+1}=x^{k+1}-x^{k}
$$

770 is approximated to find $x^{k+1}$. Here, $x^{n+1(\omega+1)(k)}$ is considered a constant.

Abaqus requires the quantities:

$$
\boldsymbol{d}_{\varepsilon}^{r}=\frac{\partial\left(\delta r^{p l}\right)}{\partial(\boldsymbol{\delta} \varepsilon)}
$$

and

$$
d_{T}^{r}=\frac{\partial\left(\delta r^{p l}\right)}{\partial(\delta T)}
$$

which, at the end of a $\omega$ loop, must satisfy the equation:

$$
\delta r^{p l}=\boldsymbol{d}_{\varepsilon}^{r}: \boldsymbol{\delta} \varepsilon+d_{T}^{r} \cdot \delta T
$$

Here, $r^{p l}$ is the quantity of energy rate found in equation 116 :

$$
r^{p l}=C_{v} \dot{T}-\dot{Q}
$$

To this end, we start with the expression from 114):

$$
r^{p l}=C_{v} \dot{T}-\dot{Q}=\sum_{j}\left\{\left[\pi^{j}-\left[\frac{\partial s_{o}}{\partial p^{j}}+\boldsymbol{\sigma}: \frac{\partial \boldsymbol{\alpha}}{\partial p^{j}}+\frac{\partial C_{v}}{\partial p^{j}} \ln \left(\frac{T}{T_{o}}\right)\right] T\right] \dot{p}^{j}\right\}-\dot{\boldsymbol{\sigma}}: \boldsymbol{\alpha} \cdot T
$$

The following definition is given:

$$
\bar{\pi}^{j}=\left[\frac{\partial s_{o}}{\partial p^{j}}+\boldsymbol{\sigma}: \frac{\partial \boldsymbol{\alpha}}{\partial p^{j}}+\frac{\partial C_{v}}{\partial p^{j}} \ln \left(\frac{T}{T_{o}}\right)\right] T
$$

The differential $\delta r^{p l}$ is approximated:

$$
\delta r^{p l}=\delta\left\{\sum_{j}\left[\left(\pi^{j}-\bar{\pi}^{j}\right) \dot{p}^{j}\right]-\dot{\boldsymbol{\sigma}}: \boldsymbol{\alpha} \cdot T\right\}
$$

where the rate variables are substituted with the finite differences of the $\mathrm{n}$ loop:

$$
\delta r^{p l}=\delta\left\{\sum_{j}\left(\left(\pi^{j}-\bar{\pi}^{j}\right) \frac{\Delta p^{j}}{\Delta t}\right)-\frac{\boldsymbol{\Delta} \boldsymbol{\sigma}}{\Delta t}: \boldsymbol{\alpha} \cdot T\right\}
$$


This differential operation yields:

$$
\begin{aligned}
& \delta r^{p l}=\delta\left\{\sum_{j}\left[\left(\pi^{j}-\bar{\pi}^{j}\right) \frac{\Delta p^{j}}{\Delta t}\right]-\frac{\boldsymbol{\Delta} \boldsymbol{\sigma}}{\Delta t}: \boldsymbol{\alpha} \cdot T\right\}= \\
& =\frac{1}{\Delta t}\left\{\sum_{j}\left[\left(\delta \pi^{j}-\delta \bar{\pi}^{j}\right) \Delta p^{j}+\left(\pi^{j}-\bar{\pi}^{j}\right) \delta p^{j}\right]-(\boldsymbol{\delta} \boldsymbol{\sigma}: \boldsymbol{\alpha} \cdot T+\boldsymbol{\Delta} \boldsymbol{\sigma}: \boldsymbol{\delta} \boldsymbol{\alpha} \cdot T+\boldsymbol{\Delta} \boldsymbol{\sigma}: \boldsymbol{\alpha} \cdot \delta T)\right\}
\end{aligned}
$$

where $\Delta t$ is the time increment corresponding to step $\mathrm{n}$.

According to convex cutting plane considerations, the various differentials appearing in the last equation are:

$$
\delta \pi^{j}=\delta \sum_{i}\left(A_{i}^{j}: \Lambda_{i}^{j}\right)=\sum_{i}\left(\delta A_{i}^{j}: \Lambda_{i}^{j}\right)
$$

Here,

$$
\begin{aligned}
\boldsymbol{\delta} \boldsymbol{A}_{\boldsymbol{i}}^{j} & =\frac{\partial \boldsymbol{A}_{\boldsymbol{i}}^{j}}{\partial \boldsymbol{\sigma}}: \boldsymbol{\delta} \boldsymbol{\sigma}+\frac{\partial \boldsymbol{A}_{\boldsymbol{i}}^{j}}{\partial T} \delta T+\sum_{g}\left[\sum_{f}\left(\frac{\partial \boldsymbol{A}_{\boldsymbol{i}}^{j}}{\partial \boldsymbol{V}_{\boldsymbol{f}}^{g}}: \boldsymbol{\delta} \boldsymbol{V}_{\boldsymbol{f}}^{\boldsymbol{g}}\right)\right]= \\
& =\frac{\partial \boldsymbol{A}_{\boldsymbol{i}}^{j}}{\partial \boldsymbol{\sigma}}: \boldsymbol{\delta} \boldsymbol{\sigma}+\frac{\partial \boldsymbol{A}_{\boldsymbol{i}}^{j}}{\partial T} \delta T+\sum_{g}\left[\delta p^{g}\left(\sum_{f} \frac{\partial \boldsymbol{A}_{\boldsymbol{i}}^{j}}{\partial \boldsymbol{V}_{\boldsymbol{f}}^{\boldsymbol{g}}}: \boldsymbol{\Lambda}_{\boldsymbol{f}}^{g}\right)\right]
\end{aligned}
$$

The indices $\mathrm{j}$ and $\mathrm{g}$ give reference to strain mechanisms, while the indices $\mathrm{i}$ and $\mathrm{f}$ refer to model variables associated to the strain mechanism denoted by $\mathrm{j}$ and $\mathrm{g}$ respectively.

Replacing the finding of A.18 in A.17 yields:

$$
\delta \pi^{j}=\sum_{i}\left\{\boldsymbol{\Lambda}_{\boldsymbol{i}}^{j}:\left[\frac{\partial \boldsymbol{A}_{\boldsymbol{i}}^{j}}{\partial \boldsymbol{\sigma}}: \boldsymbol{\delta} \boldsymbol{\sigma}+\frac{\partial \boldsymbol{A}_{\boldsymbol{i}}^{j}}{\partial T} \delta T+\sum_{g}\left[\delta p^{g} \sum_{f}\left(\frac{\partial \boldsymbol{A}_{\boldsymbol{i}}^{j}}{\partial \boldsymbol{V}_{\boldsymbol{f}}^{\boldsymbol{g}}}: \boldsymbol{\Lambda}_{\boldsymbol{f}}^{g}\right)\right]\right]\right\}
$$

or

$$
\delta \pi^{j}=\sum_{i}\left[\boldsymbol{\Lambda}_{\boldsymbol{i}}^{j}:\left(\frac{\partial \boldsymbol{A}_{\boldsymbol{i}}^{j}}{\partial \boldsymbol{\sigma}}: \boldsymbol{\delta} \boldsymbol{\sigma}+\frac{\partial \boldsymbol{A}_{\boldsymbol{i}}^{j}}{\partial T} \delta T\right)\right]+\sum_{g}\left(\Gamma_{g}^{j} \delta p^{g}\right)
$$

Here, for the sake of simplicity, new variables $\Gamma_{g}^{j}$ are defined to satisfy the equality:

$$
\sum_{g} \Gamma_{g}^{j} \delta p^{g}=\sum_{i}\left\{\boldsymbol{\Lambda}_{i}^{j}: \sum_{g}\left[\delta p^{g} \sum_{f}\left(\frac{\partial \boldsymbol{A}_{i}^{j}}{\partial \boldsymbol{V}_{f}^{g}}: \boldsymbol{\Lambda}_{\boldsymbol{f}}^{g}\right)\right]\right\}
$$

It is shown that:

$$
\Gamma_{g}^{j}=\sum_{i}\left[\boldsymbol{\Lambda}_{i}^{j}: \sum_{f}\left(\frac{\partial \boldsymbol{A}_{i}^{j}}{\partial \boldsymbol{V}_{f}^{g}}: \boldsymbol{\Lambda}_{f}^{g}\right)\right]
$$

Back in equation A.16), the differentials $\delta \bar{\pi}^{j}$ and $\delta \boldsymbol{\alpha}$ are written:

$$
\delta \bar{\pi}^{j}=\frac{\partial \bar{\pi}^{j}}{\partial \boldsymbol{\sigma}}: \boldsymbol{\delta} \boldsymbol{\sigma}+\frac{\partial \bar{\pi}^{j}}{\partial T} \delta T=T \frac{\partial \boldsymbol{\alpha}}{\partial p^{j}}: \boldsymbol{\delta} \boldsymbol{\sigma}+\frac{\partial C_{v}}{\partial p^{j}} \delta T+\frac{\bar{\pi}^{j}}{T} \delta T
$$


and

$$
\boldsymbol{\delta} \boldsymbol{\alpha}=\sum_{j}\left(\frac{\partial \boldsymbol{\alpha}}{\partial p^{j}} \delta p^{j}\right)
$$

The second derivatives with respect to the scalars $s^{j}$ are ignored. In the current 775 model, they are null.

Thus, A.16 is rewritten:

$$
\delta r^{p l}=\frac{1}{\Delta t}\left(\overline{\boldsymbol{d}}_{\boldsymbol{\sigma}}^{\boldsymbol{r}}: \boldsymbol{\delta} \boldsymbol{\sigma}+\bar{d}_{T}^{r} \delta T+D_{s}\right)
$$

where, according to equations A.20, A.22, A.23, and A.24,

$$
\begin{gathered}
\overline{\boldsymbol{d}}_{\boldsymbol{\sigma}}^{r}=\sum_{j}\left\{\Delta p^{j}\left[\sum_{i}\left(\frac{\partial \boldsymbol{A}_{\boldsymbol{i}}^{j}}{\partial \boldsymbol{\sigma}}: \boldsymbol{\Lambda}_{\boldsymbol{i}}^{j}\right)-T \frac{\partial \boldsymbol{\alpha}}{\partial p^{j}}\right]\right\}-\boldsymbol{\alpha} \cdot T \\
\bar{d}_{T}^{r}=\sum_{j}\left\{\Delta p^{j}\left[\sum_{i}\left(\frac{\partial \boldsymbol{A}_{\boldsymbol{i}}^{j}}{\partial T}: \boldsymbol{\Lambda}_{\boldsymbol{i}}^{j}\right)-\frac{\partial C_{v}}{\partial p^{j}}-\frac{\bar{\pi}^{j}}{T}\right]\right\}-\boldsymbol{\Delta} \boldsymbol{\sigma}: \boldsymbol{\alpha}
\end{gathered}
$$

and

$$
D_{s}=\sum_{j}\left\{\Delta p^{j} \sum_{g}\left(\Gamma_{g}^{j} \delta p^{g}\right)+\left(\pi^{j}-\bar{\pi}^{j}\right) \delta p^{j}\right\}-\boldsymbol{\Delta} \boldsymbol{\sigma}: \sum_{j}\left(\frac{\partial \boldsymbol{\alpha}}{\partial p^{j}} \delta p^{j}\right) \cdot T
$$

The quantity $D_{s}$ can be written as an expression of a single common cofactor $\delta p^{j}$ :

$$
\begin{aligned}
D_{s} & =\sum_{j}\left[\sum_{g}\left(\Delta p^{j} \Gamma_{g}^{j} \delta p^{g}\right)\right]+\sum_{j}\left[\left(\pi^{j}-\bar{\pi}^{j}\right) \delta p^{j}\right]-\sum_{j}\left(T \boldsymbol{\Delta} \boldsymbol{\sigma}: \frac{\partial \boldsymbol{\alpha}}{\partial p^{j}} \delta p^{j}\right) \\
& =\sum_{j}\left\{\delta p^{j}\left[\sum_{g}\left(\Delta p^{g} \Gamma_{j}^{g}\right)+\pi^{j}-\bar{\pi}^{j}-\boldsymbol{\Delta} \boldsymbol{\sigma}: \frac{\partial \boldsymbol{\alpha}}{\partial p^{j}} T\right]\right\}
\end{aligned}
$$

The variables $\bar{d}_{j}^{r}$ are introduced as such:

$$
\bar{d}_{j}^{r}=\sum_{g}\left(\Delta p^{g} \Gamma_{j}^{g}\right)+\pi^{j}-\bar{\pi}^{j}-\boldsymbol{\Delta} \boldsymbol{\sigma}: \frac{\partial \boldsymbol{\alpha}}{\partial p^{j}} T
$$

In light of $\mathrm{A} .29$ and $\mathrm{A} .30$, equation $\mathrm{A.25}$ is now:

$$
\delta r^{p l}=\frac{1}{\Delta t}\left[\overline{\boldsymbol{d}}_{\boldsymbol{\sigma}}^{r}: \boldsymbol{\delta} \boldsymbol{\sigma}+\bar{d}_{T}^{r} \delta T+\sum_{j}\left(\bar{d}_{j}^{r} \delta p^{j}\right)\right]
$$

Here, replacing the expressions of $d p^{j}$ and $\boldsymbol{d} \boldsymbol{\sigma}$ with the help of equations 101 and 102 respectively, it is found that:

$$
\delta r^{p l}=\frac{1}{\Delta t}\left\{\overline{\boldsymbol{d}}_{\boldsymbol{\sigma}}^{r}:(\boldsymbol{L}: \boldsymbol{\delta} \varepsilon+\boldsymbol{\Theta}: \delta T)+\bar{d}_{T}^{r} \delta T+\sum_{j}\left[\bar{d}_{j}^{r}\left(-\boldsymbol{P}_{\boldsymbol{\varepsilon}}^{j}: \boldsymbol{\delta} \varepsilon-P_{T}^{j} \delta T\right)\right]\right\}
$$


Recalling the general form of A.10, it is evident that:

$$
\boldsymbol{d}_{\varepsilon}^{r}=\frac{1}{\Delta t}\left[\overline{\boldsymbol{d}}_{\boldsymbol{\sigma}}^{r}: \boldsymbol{L}-\sum_{j}\left(\bar{d}_{j}^{r} \boldsymbol{P}_{\varepsilon}^{j}\right)\right]
$$

and

$$
d_{T}^{r}=\frac{1}{\Delta t}\left[\overline{\boldsymbol{d}}_{\boldsymbol{\sigma}}^{r}: \Theta+\bar{d}_{T}^{r}-\sum_{j}\left(\bar{d}_{j}^{r} P_{T}^{j}\right)\right]
$$

